Interfaces and Free Boundaries 16 (2014), 269-296

DOI 10.4171/IFB/320

\title{
Lakes and rivers in the landscape: A quasi-variational inequality approach
}

\author{
JOHN W. BARRETT \\ Dept. of Mathematics, Imperial College, London SW7 2AZ, UK \\ E-mail: jwb@imperial.ac.uk \\ LEONID PRIGOZHIN \\ Dept. of Solar Energy and Environmental Physics, Blaustein Institutes for Desert Research, \\ Ben-Gurion University of the Negev, Sede Boqer Campus, 84990 Israel \\ E-mail:leonid@math.bgu.ac.il
}

[Received 4 October 2013 and in revised form 26 January 2014]

\begin{abstract}
We consider an evolutionary quasi-variational inequality arising in a simplified model of a network of lakes and rivers forming upon a given relief of the Earth. We regularize this model and derive its finite element approximation, in which the water flow is confined to the mesh edges. The primal and mixed formulations of the discretized quasi-variational inequality are used in the numerical simulations. The corresponding steady state problems are also analyzed. Finally, we compare this approach to the lattice algorithms employed in geographic information systems for the automatic extraction of river networks from digital elevation data, and derive similar algorithms for our approximation.
\end{abstract}

2010 Mathematics Subject Classification: Primary 35K86, 35R35, 65K15, 86-08.

Keywords: Quasi-variational inequality, primal and mixed formulations, numerical approximation, augmented Lagrangian method, digital elevation model, river networks.

\section{Introduction}

During the last three decades a variety of methods have been proposed for the automatic extraction of drainage networks from digital elevation datasets called digital elevation models or DEMs (see, e.g., the reviews in $[15,18,21,27,30,31]$ and the references therein). Usually, although not always, the employed DEM carries relief elevations at the points of a regular equidistant grid (the raster format) and at the core of most routing methods is the basic D8 (deterministic eight-neighbor) algorithm. In this algorithm, the flow direction from each cell (grid point) is determined by the comparison between the cell's elevation and the elevations of its eight adjacent neighbors: the flow direction is the direction of the neighbor with the maximal rate of the elevation descent. The flow direction is, however, not determined by D8 in pits (the local minima of the relief) and also in flat horizontal areas. These features of DEM-represented reliefs are the main obstacle to flow-routing and extracting the realistic drainage (river channel) networks.

Typically, pits are considered spurious and, indeed, it is often the case for low resolution DEMs. Unless a depression is assumed to represent a real closed lake, its elevations are most often raised to the level of the lowest outflow. This, however, leads to the appearance of a flat area. Furthermore, although exactly flat Earth surfaces are not typical, the raster DEMs of territories without welldeveloped topographic features, interpolated from low-vertical-resolution digitized level contours (vector format), do contain many such areas. Heuristic iterative algorithms for drainage enforcement in flat regions include creating small artificial gradients, directing the flow towards the lowest 
watershed cell, etc. (see, e.g., [13, 16, 18, 21, 30]). These algorithms have been realized as efficient computational procedures in various well-developed geographic information systems (GIS) and, despite the admitted difficulty of flow routing in low-relief regions, are usually able to extract useful information about the drainage network from a DEM.

In this work we consider a continuous analogue of flow routing models, the evolutionary quasi-variational inequality (QVI) model [23], whose primal and mixed formulations determine, respectively, the lakes and the net of drainage channels forming upon a given relief. Our aim is to investigate the relation of this continuous model to the basic cellular models successfully employed for river network delineation, and the subsequent analysis, in various geographic information systems: we arrive at such a model in several approximation/discretization steps. We also study and compute the arising discrete problems.

Physically, our continuous model is very simple. Rain water is discharged from a distributed, usually uniform, source, flows downhill, and is collected into lakes at local depressions of the relief. As a lake overflows, it passes additional water along a one-dimensional river, possibly, to another lake below. The water can also leave the system through the open boundary.

Mathematically, however, the arising variational problem is complicated. First, the model [23] is a singular limit of the QVI describing sandpile surface evolution and, in transition to this limit, the material (water) flux becomes undetermined in the lakes. Second, the set of admissible functions in this QVI is determined by a discontinuous equilibrium constraint. Third, over the hill slopes the flux is singular: water, flowing towards the steepest descent, gathers into rivers, so the flux is a vectorial measure with a partially one-dimensional support. Finally, the problem can be ill-posed: in some cases, a slight local change of the relief can, in this model, lead to a significant change of the river network. An avulsion, a sudden abandonment of a river channel and forming a new watercourse, can sometimes be caused by a small reconstruction of a real landscape too. In practice, however, this only means the DEM resolution must be sufficiently high to make the river valleys noticeable.

To deal with the first complication, we replace the limiting continuous model for water by the sand model with a positive, but very small, material angle of repose. In such a model the flux is expected to be uniquely determined, while the lakes are represented by sandpiles whose slopes, although not exactly horizontal, are only slightly inclined. This also leads to a natural small-artificial-gradient solution to flow-routing in flat areas, automatically enforcing flows towards the outlets and away from higher elevation areas, which is the aim of [13] and some other lattice algorithms.

Following [5], we approximate the discontinuous equilibrium constraint by a continuous one; and we refer to [5] also for a proof of existence of a solution to the mixed formulation of this regularized QVI problem. However, numerical approximation of the flux variable based in that work on the lowest order Raviart-Thomas finite element is inconvenient for the representation of river networks, because the singular one-dimensional measures (rivers) become smeared. It would be impractical to overcome smearing by adapting the finite element mesh to a dense river network as, e.g., in a somewhat similar optimal transportation problem, where only a few transport rays have had to be approximated, see Figure 2 in [4]. To represent river networks better, here we concurrently approximate the free surface by continuous piecewise linear finite elements; and the water flux by vectorial measures having support on the union of all element edges, with the vector measure being constant on each element edge and in a direction parallel to that edge. Such a flux approximation prevents numerical smearing of singular fluxes. 
Numerically, the regularized and discretized QVI problem is solved first using an augmented Lagrangian method with splitting. Then, completing our reduction to a basic lattice model, we employ efficient lake filling, flow routing and flux accumulation algorithms, typical of the models used in GIS, for our discretization. This allows one to solve large scale steady state problems of practical interest using high resolution DEMs.

Finally, we note that a related continuous lake-and-river model, obtained as a singular limit of a nonlinear diffusion equation, was studied in [7].

The outline of this paper is as follows. As stated above, in this paper we employ a lake-and-river model derived as a limit of the QVI model for sand surface evolution [22-24]. In the next section, we briefly recall this sand evolution model, and its regularized version [5]. In Section 3, we introduce our lake-and-river evolution model, and its finite element approximation. The corresponding steady state problem is considered in Section 4. In Section 5, we state our numerical algorithms for solving the QVI and illustrate properties of the discretization employed by two numerical examples with artificial landscapes. Finally, in Section 6 we introduce, for our approximation, lake filling, flow routing and flux accumulation algorithms and solve the steady state lake-and-river problem for a real DEM of the Réunion island using a cellular-model-like approach.

\section{A model for sand surface evolution}

Let the initial support surface $w_{0}$ be defined in a bounded domain $\Omega \subset \mathbb{R}^{2}$ with a Lipschitz boundary $\partial \Omega$. We assume that $w_{0}$ belongs to $W_{0}^{1, \infty}(\Omega)$. Suppose sand is discharged onto this surface from a distributed source with a given non-negative density $f(\underline{x}, t) \in L^{2}\left(\Omega_{T}\right)$, where $\Omega_{T}:=\Omega \times(0, T)$. The evolving surface of the growing pile $w(\underline{x}, t)$ satisfies the material balance equation

$$
\partial_{t} w+\underline{\nabla} \cdot \underline{q}=f \quad \text { in } \Omega_{T}
$$

with the initial condition $w(\cdot, 0)=w_{0}(\cdot)$. Here $\underline{q}(\underline{x}, t)$ is the horizontal projection of the flux of sand pouring down the pile surface.

The surface $w$ can partly coincide with the support $w_{0}$, and should be above the support otherwise. Wherever $w(\underline{x}, t)>w_{0}(\underline{x})$ the equilibrium condition is $|\underline{\nabla} w(\underline{x}, t)| \leqslant k_{0}$, where $k_{0}=\tan \zeta \in \mathbb{R}_{>0}$ and $\zeta$ is the material angle of repose. In the coincidence set $\left\{(\underline{x}, t) \in \Omega_{T}\right.$ : $\left.w(\underline{x}, t)=w_{0}(\underline{x})\right\}$ this equilibrium condition is not applied, as the rigid support can be steeper. Therefore the equilibrium condition for a growing sandpile in this model is

$$
|\underline{\nabla} w| \leqslant M(w) \quad \text { in } \Omega_{T},
$$

where for any $\varphi \in C(\bar{\Omega})$

$$
M(\varphi)(\underline{x}):= \begin{cases}k_{0} & \varphi(\underline{x})>w_{0}(\underline{x}), \\ \max \left\{k_{0},\left|\underline{\nabla} w_{0}(\underline{x})\right|\right\} & \text { otherwise. }\end{cases}
$$

The surface flow of sand is forbidden wherever the surface is not steep enough, i.e., $\underline{q}=\underline{0}$ if $|\underline{\nabla} w|<k_{0}$. In addition, flow is allowed only in the steepest descent direction, $-\underline{\nabla} w$, so we have that $-\underline{\nabla} w \cdot \underline{q}=|\underline{\nabla} w||\underline{q}|$. As $\underline{q}$ can be nonzero only if the slope is critical, i.e., $|\underline{\nabla} w|=M(w)$, it follows that

$$
-\underline{\nabla} w \cdot \underline{q}=M(w)|\underline{q}| \quad \text { in } \Omega_{T} .
$$


Finally, we assume that material can leave the system freely through the domain boundary, so we set $w=0$ on $\partial \Omega$. Our sand model consists of the mass balance equation (2.1) supplemented by the conditions (2.2a,b), (2.3) and the stated boundary and initial conditions.

A more convenient form is a variational formulation of this model, which we now derive. The flux $q$ can be excluded, if only the free surface $w$ is required to be found. Let us define, for any $\eta \in \bar{C}(\bar{\Omega})$, the closed convex non-empty set

$$
K(\eta):=\left\{\varphi \in W_{0}^{1, \infty}(\Omega):|\underline{\nabla} \varphi| \leqslant M(\eta) \text { a.e. in } \Omega\right\} .
$$

Since $M(w)|q|+\underline{\nabla} \varphi \cdot \underline{q} \geqslant 0$ for any $\varphi \in K(w)$, we deduce from (2.3) that $\underline{\nabla}(\varphi-w) . q \geqslant 0$ a.e. in $\Omega$. Furthermore, on noting (2.2a) and that $w=0$ on $\partial \Omega$, we have that $w \in K(w)$. Hence, for a.a. $t \in(0, T)$

$$
\int_{\Omega} \underline{\nabla} \cdot \underline{q}(w-\varphi) \mathrm{d} \underline{x} \geqslant 0 \quad \forall \varphi \in K(w)
$$

and, making use of equation (2.1), we arrive at an evolutionary QVI:

Find $w(\underline{x}, t)$ such that $w(\cdot, 0)=w_{0}(\cdot)$ and for a.a. $t \in(0, T) w \in K(w)$ solves

$$
\int_{\Omega}\left(\partial_{t} w-f\right)(\varphi-w) \mathrm{d} \underline{x} \geqslant 0 \quad \forall \varphi \in K(w) .
$$

This formulation, written solely for the pile surface, we will call the primal problem. If $\left|\underline{\nabla} w_{0}\right| \leqslant k_{0}$ a.e. in $\Omega$, then problem (2.5) becomes a variational inequality $(K(w) \equiv K)$ and existence of a unique solution, $w \in L^{\infty}(0, T ; K) \cap W^{1,2}\left(0, T ; L^{2}(\Omega)\right)$, has been shown in [2, 24]. The dual variable, the surface flux $\underline{q}$, can in this case be sought in the space of vector-valued bounded Radon measures with an $L^{2}$ divergence. Numerical schemes based upon dual variational formulations written solely in terms of this variable, [3,8], enable one to compute approximations both to the evolving surface $w$ and the flux $\underline{q}$.

The QVI case is much more complicated. In this case it is less convenient to use a dual formulation of the QVI (2.5) in terms of the surface flux alone. To derive a variational formulation written for both variables, $w$ and $q$, in the QVI case, we note that (2.2a) holds if and only if $M(w)|\psi|+\underline{\nabla} w \cdot \psi \geqslant 0$ a.e. in $\Omega_{T}$ for any test field $\psi$. Replacing for a.a. $t \in(0, T)$ the relations (2.2a) and (2.3) by the equivalent variational inequality,

$$
\int_{\Omega}[M(w)(|\underline{\psi}|-|\underline{q}|)-w \underline{\nabla} \cdot(\underline{\psi}-\underline{q})] \mathrm{d} \underline{x} \geqslant 0
$$

for any sufficiently smooth test field $\psi$, we obtain the mixed variational formulation (2.1) and (2.6).

Existence of a solution for a weak formulation of a regularized version of this problem has recently been proved (under some additional assumptions on the domain and support) in [5]. Here we also use such a regularization, and consider the sand surface evolution model with a continuous operator $M_{\varepsilon}: C(\bar{\Omega}) \rightarrow C(\bar{\Omega})$, replacing the operator $M$ in the equilibrium constraint (2.2a). For a small $\varepsilon>0$, we approximate the initial data $w_{0} \in W_{0}^{1, \infty}(\Omega)$ by $w_{0, \varepsilon} \in W_{0}^{1, \infty}(\Omega) \cap C^{1}(\bar{\Omega})$, and define for any $\varphi \in C(\bar{\Omega})$

$$
M_{\varepsilon}(\varphi)(\underline{x}):= \begin{cases}k_{0} & \varphi(\underline{x}) \geqslant w_{0, \varepsilon}(\underline{x})+\varepsilon, \\ k_{1}(\underline{x})+\left(k_{0}-k_{1}(\underline{x})\right)\left(\frac{\varphi(\underline{x})-w_{0, \varepsilon}(\underline{x})}{\varepsilon}\right) & \varphi(\underline{x}) \in\left[w_{0, \varepsilon}(\underline{x}), w_{0, \varepsilon}(\underline{x})+\varepsilon\right], \\ k_{1}(\underline{x}):=\max \left\{k_{0},\left|\underline{\nabla} w_{0, \varepsilon}(\underline{x})\right|\right\} & \varphi(\underline{x}) \leqslant w_{0, \varepsilon}(\underline{x})\end{cases}
$$


in order to replace the jump of $M$ at $\varphi=w_{0}$ in (2.2b) by a continuous transition over an interval of the length $\varepsilon$. Omitting the details, see [5], we only note that there exists a weak solution $\{w, q\}$ to the regularized variational problem (2.1) and (2.6), with $M$ replaced by $M_{\varepsilon}$, such that $w$ is a weak solution to the corresponding regularized version of the primal QVI (2.5). We note that the regularization of $M$ is useful also for the numerical solution of these problems. We remark also that existence of $w$, a weak solution to this regularized version of (2.5), follows also from the recent work of Rodrigues and Santos [26].

Finally, as is noted in [5], to prevent an uncontrollable material influx into the domain $\Omega$ through its boundary $\partial \Omega$, in the QVI case we should assume that $\underline{n} \cdot \underline{\nabla} w_{0}<k_{0}\left(\right.$ or $\left.\underline{n} \cdot \underline{\nabla} w_{0, \varepsilon}<k_{0}\right)$ on $\partial \Omega$, where $\underline{n}$ is the outward unit normal to $\partial \Omega$. The boundary condition $w=0$ on $\partial \Omega$ and that solutions to $(2.5)$ are non-decreasing in time, see [5, 24], then ensure that there is no influx through the boundary also for $t>0$.

\section{Lakes and rivers: A model and its approximation}

Now let $f \in L^{2}\left(\Omega_{T}\right)$ be the precipitation rate, and rainwater, regarded as sand with zero repose angle $\left(k_{0}=0\right)$, be flowing downhill in the steepest descent directions and accumulating into lakes at local depressions of the Earth's relief. We assume that water neither penetrates the soil nor evaporates. Then both the equilibrium condition (2.2a, b) and the balance equation (2.1) remain valid.

Contrary to sandpiles $\left(k_{0}>0\right)$, the flow in the lakes is not confined to a thin surface layer and its direction is not determined as the steepest descent direction. Nevertheless, lake hydrodynamics does not affect the free surface, which is either the horizontal lake surface, $\underline{\nabla} w=\underline{0}$ for $w>w_{0}$, or coincides with the Earth's relief, $w=w_{0}$. Although the flux $q$ in the lakes is not determined by our model uniquely, the degenerate $\left(k_{0}=0\right)$ primal QVI $(2.5)$ still describes the free surface evolution, see [23]. This inequality (or its regularized version) can be used to find the lake areas. It is, however, the water flux in the coincidence set $w=w_{0}$, which is usually the main interest. The drainage (river) network is defined as the subset of $\Omega$ in which $|q| \geqslant q_{0}$, where $q_{0}$ is the desired resolution of a hydrological map.

Since the water flux in the lakes is not unique, it is convenient to regularize the problem further and replace $k_{0}=0$ by a small $k_{0}>0$. Lakes in this case become piles with a negligibly small slope incline. This regularization induces small artificial gradients also in flat surface areas and, therefore, leads there to a natural way of flux routing.

To calculate the water flux one could approximate the regularized mixed formulation (2.1) and (2.6), with $M$ replaced by $M_{\varepsilon}$, using the divergence conforming Raviart-Thomas elements of the lowest order for the flux $q$ and piecewise constants for the free surface $w$ (see [5]). Such an approach, however, would lead to a "smeared" representation of the singular water flux in the rivers, inhibiting the delineation of rivers, and hence the derivation of hydrological maps. This makes this representation inconvenient also for the analysis of river basins and for computing hydrological characteristics of drainage networks. In this work we present a hydrology-oriented alternative: a discrete approximation of the regularized model (2.1) and (2.6) in which rainwater flows through a network of drainage channels.

Let $\Omega^{h}$ be a polygonal approximation of $\Omega$, and $\mathcal{T}^{h}$ be a regular partitioning of $\Omega^{h}$ into triangles $\sigma$ so that

$$
\Omega^{h}=\bigcup_{\sigma \in \mathcal{T}^{h}} \bar{\sigma} \quad \text { with } \quad h:=\max _{\sigma \in \mathcal{T}^{h}} \operatorname{diam}(\sigma) .
$$


We assume that the vertices of $\mathcal{T}^{h}$ lying on $\partial \Omega^{h}$, the boundary of $\Omega^{h}$, also lie on $\partial \Omega$. Let $\mathcal{V}^{h}$ and $\mathcal{E}^{h}$ be the sets of vertices and oriented edges of $\mathcal{T}^{h}$, respectively. The edge $e_{k, j} \in \mathcal{E}^{h}$ is determined by two neighboring vertices, $\underline{v}_{k}, \underline{v}_{j} \in \mathcal{V}^{h}$ and is oriented from $\underline{v}_{k}$ to $\underline{v}_{j}$. In addition, $\underline{i}_{e}$ denotes the unit vector in the direction of edge $e \in \mathcal{E}^{h}$. We define $\mathcal{V}_{I}^{h}:=\mathcal{V}^{h} \backslash \mathcal{V}_{B}^{h}$ and $\mathcal{E}_{I}^{h}:=\mathcal{E}^{h} \backslash \mathcal{E}_{B}^{h}$, where the subscripts $B$ and $I$ denote "boundary" and "internal", respectively. So an edge $e \in \mathcal{E}_{B}^{h}$ is such that $e \subset \partial \Omega^{h}$. Let $C_{0}\left(\Omega^{h}\right)$ denote continuous functions on $\Omega^{h}$, which vanish on the boundary. We then set

$$
\begin{aligned}
& U_{0}^{h}:=\left\{\varphi \in C_{0}\left(\Omega^{h}\right):\left.\varphi\right|_{\sigma} \text { is linear } \forall \sigma \in \mathcal{T}^{h}\right\}, \quad \text { and } \\
& \underline{V}^{h}:=\left\{\underline{\psi} \in\left[\mathcal{M}\left(\Omega^{h}\right)\right]^{2}: \underline{\psi}=\sum_{e \in \mathcal{E}^{h}} \psi_{e} \underline{i}_{e} \mathrm{~d} \mathcal{H}^{1}(e)\right\},
\end{aligned}
$$

where $\psi_{e} \in \mathbb{R}, d \mathcal{H}^{1}(e)$ is the one-dimensional Hausdorff measure supported on edge $e$, and $\mathcal{M}\left(\Omega^{h}\right)$ is the Banach space of bounded Radon measures; that is, $\mathcal{M}\left(\Omega^{h}\right):=\left[C\left(\Omega^{h}\right)\right]^{*}$, the dual of $C\left(\Omega^{h}\right)$. The duality pairing between $\mathcal{M}\left(\Omega^{h}\right)$ and $C\left(\Omega^{h}\right)$ is denoted by $\langle\cdot, \cdot\rangle$, and is naturally extended to vectors so that

$$
\langle\underline{\psi}, \underline{\phi}\rangle=\sum_{e \in \mathcal{E}^{h}} \psi_{e} \underline{i}_{e} \cdot \int_{e} \underline{\phi} \mathrm{d} e \quad \forall \underline{\psi} \in \underline{V}^{h}, \underline{\phi} \in\left[C\left(\Omega^{h}\right)\right]^{2} .
$$

We denote by $(\cdot, \cdot)$ the standard inner product on $\Omega^{h}$. We introduce also for all $\varphi, \eta \in U_{0}^{h}$

$$
(\varphi, \eta)^{h}:=\sum_{\sigma \in \mathcal{T}^{h}}(\varphi, \eta)_{\sigma}^{h}, \quad \text { where } \quad(\varphi, \eta)_{\sigma}^{h}:=\frac{1}{3}|\sigma| \sum_{j=1}^{3} \varphi\left(\underline{v}_{j}^{(\sigma)}\right) \eta\left(\underline{v}_{j}^{(\sigma)}\right)
$$

with $|\sigma|$ and $\left\{\underline{v}_{j}^{(\sigma)}\right\}_{j=1}^{3}$ being the area and vertices of $\sigma$.

Let $W^{0} \in U_{0}^{h}$ be such that $W^{0}\left(\underline{v}_{k}\right)=w_{0}\left(\underline{v}_{k}\right)$ for all $\underline{v}_{k} \in \mathcal{V}^{h}$, where $w_{0} \in W_{0}^{1, \infty}(\Omega)$ is the Earth's relief on $\bar{\Omega}$. Below, in some cases it will be convenient to allow for some water initially upon the support surface $W^{0}$, so we assume the initial condition $W^{1} \in U_{0}^{h}$ is given and satisfies $W^{1} \geqslant W^{0}$.

The continuous piecewise linear representation of the relief, employed in our approximation, is called a "triangulated irregular network" format (TIN) in the geographic literature, see, e.g., [17]. TIN DEMs can use different resolution in different parts of the domain, and so ensure accurate surface representation using less sampling points than the raster DEMs. The disadvantage of using such a format though is the need for less efficient and more complicated flow routing algorithms. However, since, as in landscape evolution models [6, 25], the flow in our model is a priori confined to the mesh edges, simple but efficient algorithms for lake filling, flow routing and flux accumulation can be proposed, see Section 6 for details.

On partitioning $[0, T]$ into possibly variable time steps $\tau_{n}:=t_{n}-t_{n-1}, n=2, \ldots, N$, and defining $\tau:=\max _{n=2, \ldots, N} \tau_{n}$ and

$$
f^{n}(\cdot):=\frac{1}{\tau_{n}} \int_{t_{n-1}}^{t_{n}} f(\cdot, t) \mathrm{d} t \in L^{2}(\Omega), \quad n=2, \ldots, N ;
$$

we then consider, as an approximation to (2.1) and (2.6) for $0<k_{0} \ll 1$, the finite-dimensional problem: 
(Q) For $n=2, \ldots, N$, find $W^{n} \in U_{0}^{h}$ and $\underline{Q}^{n} \in \underline{V}^{h}$ such that

$$
\begin{aligned}
\left(\frac{W^{n}-W^{n-1}}{\tau_{n}}, \varphi\right)^{h}-\left\langle\underline{Q}^{n}, \underline{\nabla} \varphi\right\rangle=\left(\widetilde{f}^{n}, \varphi\right) & \forall \varphi \in U_{0}^{h}, \\
\left\langle|\underline{\psi}|-\left|\underline{Q}^{n}\right|, M_{\varepsilon}^{h}\left(W^{n}\right)\right\rangle+\left\langle\underline{\psi}-\underline{Q}^{n}, \underline{\nabla} W^{n}\right\rangle \geqslant 0 & \forall \underline{\psi} \in \underline{V}^{h},
\end{aligned}
$$

where $\widetilde{f}^{n} \in L^{2}\left(\Omega^{h}\right)$ is a nonnegative extension of $f^{n}$ from $\Omega$ to $\Omega^{h}$, if $\Omega^{h} \not \Omega$. In addition, the approximation $M_{\varepsilon}^{h}$ of the operator $M_{\varepsilon}$, which is a constant $\left(\geqslant k_{0}\right)$ on any edge, will be defined below. Although $\underline{\nabla} \varphi$ does not belong to $\left[C\left(\Omega^{h}\right)\right]^{2},\langle\psi, \underline{\nabla} \varphi\rangle$ is well-defined for any $\psi \in \underline{V}^{h}$ and any $\varphi \in U_{0}^{h}$, because the scalar product $\underline{i}_{e} \cdot \underline{\nabla} \varphi$ is continuous across any edge $e \in \mathcal{E}_{I}^{h}$.

Let $\chi_{j} \in U_{0}^{h}$ be the standard hat basis function associated with vertex $\underline{v}_{j} \in \mathcal{V}_{I}^{h}$; that is, $\chi_{j}\left(\underline{v}_{k}\right)=$ $\delta_{j k}$ for all $\underline{v}_{k} \in \mathcal{V}_{I}^{h}$. Then for any $\varphi \in U_{0}^{h}$, we can write

$$
\varphi(\underline{x})=\sum_{\underline{v}_{j} \in \mathcal{V}_{I}^{h}} \varphi_{j} \chi_{j}(\underline{x}), \quad \text { where } \varphi_{j}=\varphi\left(\underline{v}_{j}\right) .
$$

For any $\underline{\psi} \in \underline{V}^{h}$, it follows from (3.2) that

$$
\left\langle\underline{\psi}, \underline{\nabla} \chi_{j}\right\rangle=\sum_{e=e_{k, j} \in \mathcal{E}_{I}^{h}} \psi_{e}-\sum_{e=e_{j, k} \in \mathcal{E}_{I}^{h}} \psi_{e}=\sum_{e_{k, j} \in \mathcal{E}_{I}^{h}} \psi_{e_{k, j}}-\sum_{e_{j, k} \in \mathcal{E}_{I}^{h}} \psi_{e_{j, k}} .
$$

On setting

$$
s_{j}:=\left(\chi_{j}, \chi_{j}\right)^{h}=\int_{\Omega^{h}} \chi_{j} \mathrm{~d} \underline{x}>0 \quad \text { and } \quad F_{j}^{n}:=\frac{1}{s_{j}} \int_{\Omega^{h}} \widetilde{f}^{n} \chi_{j} \mathrm{~d} \underline{x} \geqslant 0 \quad \forall \underline{v}_{j} \in \mathcal{V}_{I}^{h},
$$

and noting (3.5) and (3.6), we can rewrite (3.4a) as

$$
s_{j} \frac{W_{j}^{n}-W_{j}^{n-1}}{\tau_{n}}+\sum_{e_{j, k} \in \mathcal{E}_{I}^{h}} Q_{e_{j, k}}^{n}-\sum_{e_{k, j} \in \mathcal{E}_{I}^{h}} Q_{e_{k, j}}^{n}=s_{j} F_{j}^{n} \quad \forall \underline{v}_{j} \in \mathcal{V}_{I}^{h} .
$$

Choosing $\psi$ in (3.4b) such that $\psi_{e}=Q_{e}^{n}$ for all edges $e$ except one edge $e_{k, j} \in \mathcal{E}^{h}$ we obtain, on noting (3.2) and (3.6), that

$$
\left.\left(|\psi|-\left|Q_{e_{k, j}}^{n}\right|\right)\left|e_{k, j}\right| M_{\varepsilon}^{h}\left(W^{n}\right)\right|_{e_{k, j}}+\left(\psi-Q_{e_{k, j}}^{n}\right)\left(W_{j}^{n}-W_{k}^{n}\right) \geqslant 0 \quad \forall \psi \in \mathbb{R} \quad \forall e_{k, j} \in \mathcal{E}^{h},
$$

where $|e|$ is the length of edge $e$. Choosing $\psi=0$ and $\psi=2 Q_{e_{k, j}}^{n}$ in (3.8b) yields that it is equivalent to

$$
\begin{aligned}
&\left.\left|Q_{e_{k, j}}^{n}\right|\left|e_{k, j}\right| M_{\varepsilon}^{h}\left(W^{n}\right)\right|_{e_{k, j}}+Q_{e_{k, j}}^{n}\left(W_{j}^{n}-W_{k}^{n}\right)=0 \quad \text { and } \\
&\left.|\psi|\left|e_{k, j}\right| M_{\varepsilon}^{h}\left(W^{n}\right)\right|_{e_{k, j}}+\psi\left(W_{j}^{n}-W_{k}^{n}\right) \geqslant 0 \quad \forall \psi \in \mathbb{R} \quad \forall e_{k, j} \in \mathcal{E}^{h} .
\end{aligned}
$$

It remains to define our edge approximation $\left.M_{\varepsilon}^{h}\right|_{e_{k, j}}, e_{k, j} \in \mathcal{E}^{h}$, of the operator $M_{\varepsilon}$.

In the regularized continuous QVI problem the inequality $|\underline{\nabla} w| \leqslant M_{\varepsilon}(w)$ holds a.e.; and flow is only allowed where $|\underline{\nabla} w|=M_{\varepsilon}(w)$, and is in the direction of steepest descent. We would like a 
similar behavior for our network approximation $(3.8 \mathrm{a}, \mathrm{b})$. However, in the network case the steepest slope of surface descent along the edges generally differs from the steepest slope of ascent along the edges from the same vertex. Only the slope of descent is important, and we will allow a nonzero edge flux $Q_{e}^{n}, e \in \mathcal{E}^{h}$, only if all of the following conditions hold:

(i) from the vertex of edge $e$ with a higher $W^{n}$ value;

(ii) if for this vertex the edge $e$ is the edge of steepest descent;

(iii) if the surface slope along this edge is "critical", i.e., $\left|\partial_{\underline{i}_{e}} W^{n}\right|=M_{\varepsilon}^{h}\left(W^{n}\right)$ on $e$,

where $\underline{\partial}_{\underline{i_{e}}} W^{n}:=\underline{i}_{e} \cdot \underline{\nabla} W^{n}$ and $\left.M_{\varepsilon}^{h}\right|_{e}$ is still to be defined.

If a solution exists to $(\mathrm{Q}),(3.4 \mathrm{a}, \mathrm{b}) \equiv(3.8 \mathrm{a}, \mathrm{b})$, then, on noting $(3.9)$, it follows that

$$
\left|\partial_{\underline{i}_{e}} W^{n}\right| \leqslant M_{\varepsilon}^{h}\left(W^{n}\right) \text { on } e \quad \forall e \in \mathcal{E}^{h}, \quad n=2, \ldots, N .
$$

Furthermore, the flux $Q_{e}^{n}$ can be nonzero only if $\left|\partial_{\underline{i}_{e}} W^{n}\right|=\left.M_{\varepsilon}^{h}\left(W^{n}\right)\right|_{e}$ and, in this case, $Q_{e}^{n}$ is positive (negative) if the flow is in the direction $\underline{i}_{e}\left(-\underline{i}_{e}\right)$. Taking this into account, we define our approximation $\left.M_{\varepsilon}^{h}\right|_{e}$ for all edges $e \in \mathcal{E}^{h}$ in two steps.

First, we define for any $\varphi \in U_{0}^{h}$ the steepest edge descent at each vertex $\underline{v}_{k} \in \mathcal{V}^{h}$ as

$$
\partial_{\downarrow}^{h} \varphi\left(\underline{v}_{k}\right):=\max \left\{\frac{\varphi_{k}-\varphi_{j}}{|e(k, j)|}: e(k, j)=e_{k, j} \in \mathcal{E}^{h} \text { or } e(k, j)=e_{j, k} \in \mathcal{E}^{h}\right\} .
$$

Then similarly to (2.7), but using $W^{0}$ instead of $w_{0, \varepsilon}$ and replacing $\left|\underline{\nabla} w_{0, \varepsilon}\left(\underline{v}_{k}\right)\right|$ by $\partial_{\downarrow}^{h} W^{0}\left(\underline{v}_{k}\right)$, we compute, for all $\underline{v}_{k} \in \mathcal{V}^{h}$, the vertex value

$$
M_{\varepsilon}^{h}(\varphi)\left(\underline{v}_{k}\right)= \begin{cases}k_{0} & \varphi_{k} \geqslant W_{k}^{0}+\varepsilon, \\ k_{1}\left(\underline{v}_{k}\right)+\left(k_{0}-k_{1}\left(\underline{v}_{k}\right)\right)\left(\frac{\varphi_{k}-W_{k}^{0}}{\varepsilon}\right) & \varphi_{k} \in\left[W_{k}^{0}, W_{k}^{0}+\varepsilon\right], \\ k_{1}\left(\underline{v}_{k}\right):=\max \left\{k_{0}, \partial_{\downarrow}^{h} W^{0}\left(\underline{v}_{k}\right)\right\} & \varphi_{k} \leqslant W_{k}^{0} .\end{cases}
$$

Second, for each edge $e_{k, j} \in \mathcal{E}^{h}$, we set the $e d g e$ value

$$
\left.M_{\varepsilon}^{h}(\varphi)\right|_{e_{k, j}}= \begin{cases}M_{\varepsilon}^{h}(\varphi)\left(\underline{v}_{k}\right) & \varphi_{k} \geqslant \varphi_{j} \\ M_{\varepsilon}^{h}(\varphi)\left(\underline{v}_{j}\right) & \text { otherwise. }\end{cases}
$$

We note that for any $\varphi, \eta \in U_{0}^{h}$ and any $\underline{v}_{k} \in \mathcal{V}^{h}$ that

$$
0<k_{0} \leqslant M_{\varepsilon}^{h}(\varphi)\left(v_{k}\right) \leqslant M_{\varepsilon}^{h}(\eta)\left(v_{k}\right) \quad \text { if } \quad \eta_{k} \leqslant \varphi_{k} .
$$

Our discrete network model $(\mathrm{Q}),(3.4 \mathrm{a}, \mathrm{b}) \equiv(3.8 \mathrm{a}, \mathrm{b})$, is now fully defined and satisfies the desired conditions (3.10) above. It follows from the equation in (3.9) as $W^{n} \in U_{0}^{h}$, (3.13) and (3.14) that

$$
\underline{Q}_{e}^{n}=\underline{0} \quad \forall e \in \mathcal{E}_{B}^{h}, \quad n=2, \ldots, N .
$$

Associated with (Q) is the following discrete analogue of the primal QVI (2.5): 
(P) For $n=2, \ldots, N$, find $W^{n} \in K^{h}\left(W^{n}\right)$ such that

$$
\left(\frac{W^{n}-W^{n-1}}{\tau_{n}}, \varphi-W^{n}\right)^{h} \geqslant\left(\widetilde{f}^{n}, \varphi-W^{n}\right) \quad \forall \varphi \in K^{h}\left(W^{n}\right),
$$

where for any $\eta \in U_{0}^{h}$

$$
\left.K^{h}(\eta):=\left\{\varphi \in U_{0}^{h}: \partial_{\downarrow}^{h} \varphi\left(\underline{v}_{j}\right) \leqslant M_{\varepsilon}^{h}(\eta) \underline{v}_{j}\right) \quad \forall \underline{v}_{j} \in \mathcal{V}^{h}\right\}
$$

is a closed convex set, and is non-empty as $\varphi^{0} \equiv 0 \in K^{h}(\eta)$. We note that $W^{0} \in K^{h}\left(W^{0}\right)$. Adopting the notation (3.5) and (3.7), we can rewrite (3.16) as

$$
\sum_{\underline{v}_{j} \in \mathcal{V}_{I}^{h}} s_{j}\left(\frac{W_{j}^{n}-W_{j}^{n-1}}{\tau_{n}}-F_{j}^{n}\right)\left(\varphi_{j}-W_{j}^{n}\right) \geqslant 0 \quad \forall \varphi \in K^{h}\left(W^{n}\right) .
$$

For future developments, we note that the inequality constraints $\left.\partial_{\downarrow}^{h} \varphi\left(\underline{v}_{j}\right) \leqslant M_{\varepsilon}^{h}(\eta) \underline{v}_{j}\right)$ for all $\underline{v}_{j} \in \mathcal{V}^{h}$ appearing in the definition (3.17) are equivalent to the edge set of inequality pairs:

$$
\varphi_{\ell}-\varphi_{k} \leqslant\left|e_{k, \ell}\right| M_{\varepsilon}^{h}(\eta)\left(\underline{v}_{\ell}\right) \quad \text { and } \quad \varphi_{k}-\varphi_{\ell} \leqslant\left|e_{k, \ell}\right| M_{\varepsilon}^{h}(\eta)\left(\underline{v}_{k}\right) \quad \forall e_{k, \ell} \in \mathcal{E}_{I}^{h} .
$$

We note that the constraints in (3.19) are automatically satisfied for $e_{k, \ell} \in \mathcal{E}_{B}^{h}$, as $\varphi \in U_{0}^{h}$.

In the following theorems and lemmas we prove existence of a solution to the primal QVI (P) and the mixed formulation $(\mathrm{Q})$, and show their equivalence. First we prove a useful lemma.

Lemma 3.1 (i) For any $\eta \in U_{0}^{h}$ we note that

$$
K^{h}(\eta) \subseteq \mathcal{B}^{h}:=\left\{\varphi \in U_{0}^{h}:|\varphi| \leqslant \mathcal{D}^{h} \text { in } \Omega^{h}\right\},
$$

where $\mathcal{D}^{h} \in U_{0}^{h}$ is such that

$$
\mathcal{D}_{j}^{h}=d^{h}\left(\underline{v}_{j}\right) \max \left\{k_{0},\left|\underline{\nabla} W^{0}\right|_{0, \infty, \Omega^{h}}\right\} \quad \forall \underline{v}_{j} \in \mathcal{V}^{h}
$$

with $\left.d^{h} \underline{v}_{j}\right)$ being the length of the shortest edge path from $\underline{v}_{j} \in \mathcal{V}^{h}$ to $\partial \Omega^{h}$.

(ii) Let $\eta^{(i)} \in K^{h}\left(\eta^{(i)}\right), i=1$, 2. Then $\eta^{\star} \in U_{0}^{h}$ such that

$$
\eta_{j}^{\star}=\max \left\{\eta_{j}^{(1)}, \eta_{j}^{(2)}\right\} \quad \forall \underline{v}_{j} \in \mathcal{V}^{h} \Rightarrow \eta^{\star} \in K^{h}\left(\eta^{\star}\right) \subseteq K^{h}\left(\eta^{(i)}\right), \quad i=1,2 .
$$

Proof. (i) The desired result (3.20a,b) follows immediately from (3.17), (3.11) and (3.12).

(ii) Similarly, on noting (3.11) and (3.12), we have for any $\underline{v}_{j} \in \mathcal{V}^{h}$ and $i=1$ or 2 that

$$
\eta_{j}^{\star}=\eta_{j}^{(i)} \Rightarrow \partial_{\downarrow}^{h} \eta^{\star}\left(\underline{v}_{j}\right) \leqslant \partial_{\downarrow}^{h} \eta^{(i)}\left(\underline{v}_{j}\right) \leqslant M_{\varepsilon}^{h}\left(\eta^{(i)}\right)\left(\underline{v}_{j}\right)=M_{\varepsilon}^{h}\left(\eta^{\star}\right)\left(\underline{v}_{j}\right) .
$$

Hence $\eta^{\star} \in K^{h}\left(\eta^{\star}\right)$. Then noting that $\eta^{*} \geqslant \eta^{(i)}, i=1,2$, (3.14) and (3.17) yield the desired result (3.21).

Theorem 3.2 Let $W^{0} \leqslant W^{1} \in K^{h}\left(W^{1}\right)$. Then there exists a solution $\left\{W^{n}\right\}_{n=2}^{N}$ to (P), (3.16) $\equiv$ (3.18), and $W^{1} \leqslant W^{2} \leqslant \ldots \leqslant W^{N}$. 
Proof. To prove existence of a solution to (P), we will apply the Brouwer fixed point theorem. We introduce the mapping $\Upsilon: U_{0}^{h} \rightarrow U_{0}^{h}$ such that given $\eta \in U_{0}^{h}, \Upsilon \eta \in K^{h}(\eta)$ is the unique solution of the convex minimization problem

$$
\min _{\varphi \in K^{h}(\eta)} G^{n}(\varphi)
$$

where the quadratic functional

$$
\begin{aligned}
G^{n}(\varphi) & :=\frac{1}{2 \tau_{n}}(\varphi, \varphi)^{h}-\frac{1}{\tau_{n}}\left(W^{n-1}, \varphi\right)^{h}-\left(\widetilde{f}^{n}, \varphi\right) \\
& =\sum_{\underline{v}_{j} \in \mathcal{V}_{I}^{h}} s_{j}\left(\frac{1}{2 \tau_{n}}\left(\varphi_{j}\right)^{2}-\left[\frac{1}{\tau_{n}} W_{j}^{n-1}+F_{j}^{n}\right] \varphi_{j}\right) .
\end{aligned}
$$

It follows from (3.20a) that $\Upsilon: \mathcal{B}^{h} \rightarrow \mathcal{B}^{h}$. Therefore to apply the Brouwer fixed point theorem we just need to show that $\Upsilon$ is continuous, as $\mathcal{B}^{h}$ is a bounded finite dimensional convex set.

Let $\left\{\eta^{(i)}\right\}_{i \in \mathbb{N}}$ be such that $\eta^{(i)} \in \mathcal{B}^{h}$ and $\eta^{(i)} \rightarrow \eta \in \mathcal{B}^{h}$ as $i \rightarrow \infty$. Then for all $i \in \mathbb{N}$, $\Upsilon \eta^{(i)} \in K^{h}\left(\eta^{(i)}\right)$ is the unique solution of the convex minimization problem (3.23) with $\eta$ replaced by $\eta^{(i)}$, and so is the unique solution of the corresponding variational inequality

$$
\left(\Upsilon \eta^{(i)}-W^{n-1}, \varphi-\Upsilon \eta^{(i)}\right)^{h} \geqslant \tau_{n}\left(\widetilde{f}^{n}, \varphi-\Upsilon \eta^{(i)}\right) \quad \forall \varphi \in K^{h}\left(\eta^{(i)}\right) .
$$

As $\Upsilon \eta^{(i)} \in \mathcal{B}^{h}, \forall i \in \mathbb{N}$, there exists $\xi^{\star} \in \mathcal{B}^{h}$ and a subsequence $\left\{\Upsilon \eta^{\left(i_{m}\right)}\right\}_{i_{m} \in \mathbb{N}}$ such that $\Upsilon_{\left.\eta^{(i m}\right)} \rightarrow$ $\xi^{\star}$ as $i_{m} \rightarrow \infty$. On noting that $M_{\varepsilon}^{h}(\cdot)\left(\underline{v}_{k}\right)$ for all $\underline{v}_{k} \in \mathcal{V}^{h}$ is continuous, recall (3.12), we have that if $\varphi \in K^{h}(\eta)$ then for all $i \in \mathbb{N}$

$$
\begin{aligned}
\varphi^{(i)} & :=\left(1+\delta^{(i)}\right)^{-1} \varphi \in K^{h}\left(\eta^{(i)}\right), \\
\text { where } \quad \delta^{(i)} & :=\max _{\underline{v}_{k} \in \mathcal{V}^{h}} \frac{\left|M_{\varepsilon}^{h}(\eta)\left(\underline{v}_{k}\right)-M_{\varepsilon}^{h}\left(\eta^{(i)}\right)\left(\underline{v}_{k}\right)\right|}{k_{0}} \rightarrow 0 \quad \text { as } \quad i \rightarrow \infty .
\end{aligned}
$$

Hence, on replacing $\varphi$ in (3.25) by $\varphi^{(i)}$, we can pass to the limit $i_{m} \rightarrow \infty$ for the subsequence in (3.25) to obtain that $\xi^{\star} \in K^{h}(\eta)$ satisfies

$$
\left(\xi^{\star}-W^{n-1}, \varphi-\xi^{\star}\right)^{h} \geqslant \tau_{n}\left(\widetilde{f}^{n}, \varphi-\xi^{\star}\right) \quad \forall \varphi \in K^{h}(\eta)
$$

Hence $\xi^{\star}=\Upsilon \eta$, and as this is the unique solution of (3.27) the whole sequence $\Upsilon_{\eta}^{(i)} \rightarrow \Upsilon_{\eta}$ as $i \rightarrow \infty$. Therefore the mapping $\Upsilon: \mathcal{B}^{h} \rightarrow \mathcal{B}^{h}$ is continuous, and so the Brouwer fixed point theorem yields that it has a fixed point $W^{n}$. Hence there exists a solution $\left\{W^{n}\right\}_{n=2}^{N}$ to (P), (3.16) $\equiv$ (3.18).

We now show that $W^{n} \geqslant W^{n-1}, n=2, \ldots, N$. Let $\varphi^{\star} \in U_{0}^{h}$ be such that $\varphi_{k}^{\star}=W_{k}^{n}+$ $\left[W_{k}^{n-1}-W_{k}^{n}\right]_{+}=\max \left\{W_{k}^{n}, W_{k}^{n-1}\right\}$ for all $\underline{v}_{k} \in \mathcal{V}^{h}$, where $[a]_{+}:=\max \{a, 0\}$ for all $a \in \mathbb{R}$. As $W^{n-1} \in K^{h}\left(W^{n-1}\right)$ and $W^{n} \in K^{h}\left(W^{n}\right)$, it follows from (3.21) that $\varphi^{\star} \in K^{h}\left(W^{n}\right)$. Choosing $\varphi=\varphi^{\star}$ in (3.18), and noting (3.7), yields that

$$
\sum_{\underline{v}_{j} \in \mathcal{E}_{I}^{h}} s_{j}\left[W_{j}^{n-1}-W_{j}^{n}\right]_{+}^{2} \leqslant-\tau_{n} \sum_{\underline{v}_{j} \in \mathcal{E}_{I}^{h}} s_{j} F_{j}^{n}\left[W_{j}^{n-1}-W_{j}^{n}\right]_{+} \leqslant 0
$$

and hence $W^{n} \geqslant W^{n-1}$. 
For the next result, we introduce

$$
\begin{aligned}
& \mathcal{A}^{h}:=\left\{\psi:\left\{\psi_{k, \ell}\right\}_{e_{k, \ell} \in \mathcal{E}_{I}^{h}} \text { with } \psi_{k, \ell} \in \mathbb{R}\right\}, \\
& \mathcal{A}_{\geqslant 0}^{h}:=\left\{\psi \in \mathcal{A}^{h}: \psi_{k, \ell} \in \mathbb{R}_{\geqslant 0} \quad \forall e_{k, \ell} \in \mathcal{E}_{I}^{h}\right\} .
\end{aligned}
$$

Existence of a solution to the mixed formulation (Q) is a consequence of Theorem 3.2 and the following equivalence result.

THEOREM 3.3 If $\left\{W^{n}\right\}_{n=2}^{N}$ solves (P), then there exists $\left\{\underline{Q}^{n}\right\}_{n=2}^{N}$ such that $\left\{W^{n}, \underline{Q}^{n}\right\}_{n=2}^{N}$ solves (Q). Hence there exists a solution $\left\{W^{n}, \underline{Q}^{n}\right\}_{n=2}^{N}$ to $(\mathrm{Q}),(3.4 \mathrm{a}, \mathrm{b}) \equiv(3.8 \mathrm{a}, \mathrm{b})$.

Proof. Let $\left\{W^{n}\right\}_{n=2}^{N}$ solve (P), (3.16) $\equiv$ (3.18). Then $\varphi=W^{n}$ is the unique solution to the following convex minimization problem

$$
\min _{\varphi \in K^{h}\left(W^{n}\right)} G^{n}(\varphi)
$$

where $G^{n}(\cdot)$ is defined by (3.24).

In the regularized model $k_{0}>0$, the Slater constraint qualification hypothesis (see, e.g., (5.34) on p. 69 in [9]) is obviously satisfied with $\varphi^{0} \equiv 0$; that is, $\partial_{\downarrow}^{h} \varphi^{0}\left(\underline{v}_{j}\right)<M_{\varepsilon}^{h}\left(W^{n}\right)\left(\underline{v}_{j}\right)$ for all $\underline{v}_{j} \in \mathcal{V}^{h}$. On recalling (3.19), we now introduce the Lagrangian

$$
\begin{aligned}
L^{n}(\varphi, \alpha, \beta):=G^{n}(\varphi) & +\sum_{e_{k, \ell} \in \mathcal{E}_{I}^{h}} \alpha_{k, \ell}\left[\varphi_{\ell}-\varphi_{k}-\left|e_{k, \ell}\right| M_{\varepsilon}^{h}\left(W^{n}\right)\left(\underline{v}_{\ell}\right)\right] \\
& +\sum_{e_{k, \ell} \in \mathcal{E}_{I}^{h}} \beta_{k, \ell}\left[\varphi_{k}-\varphi_{\ell}-\left|e_{k, \ell}\right| M_{\varepsilon}^{h}\left(W^{n}\right)\left(\underline{v}_{k}\right)\right]
\end{aligned}
$$

where $\alpha, \beta \in \mathcal{A}^{h}$. It follows from the Kuhn-Tucker theorem (see, e.g., Theorem 5.2 in [9]) that there exist Lagrange multipliers $\alpha^{\star}, \beta^{\star} \in \mathcal{A}_{\geqslant 0}^{h}$ such that $\left\{W^{n}, \alpha^{\star}, \beta^{\star}\right\}$ is a saddle point of the Lagrangian, i.e.,

$$
L^{n}\left(W^{n}, \alpha, \beta\right) \leqslant L^{n}\left(W^{n}, \alpha^{\star}, \beta^{\star}\right) \leqslant L^{n}\left(\varphi, \alpha^{\star}, \beta^{\star}\right) \quad \forall \varphi \in U_{0}^{h} \quad \forall \alpha, \beta \in \mathcal{A}_{\geqslant 0}^{h} .
$$

The first inequality in (3.30) yields that

$$
\begin{aligned}
& \alpha_{k, \ell}^{\star}\left[W_{\ell}^{n}-W_{k}^{n}-\left|e_{k, \ell}\right| M_{\varepsilon}^{h}\left(W^{n}\right)\left(\underline{v}_{\ell}\right)\right]=0 \quad \text { and } \\
& \beta_{k, \ell}^{\star}\left[W_{k}^{n}-W_{\ell}^{n}-\left|e_{k, \ell}\right| M_{\varepsilon}^{h}\left(W^{n}\right)\left(\underline{v}_{k}\right)\right]=0 \quad \forall e_{k, \ell} \in \mathcal{E}_{I}^{h} .
\end{aligned}
$$

Hence $\alpha_{k, \ell}^{\star}$ can be positive only if $W_{\ell}^{n}-W_{k}^{n}=\left|e_{k, \ell}\right| M_{\varepsilon}^{h}\left(W^{n}\right)\left(\underline{v}_{\ell}\right)$. In this case $W_{\ell}^{n}>W_{k}^{n}$, so $\beta_{k, \ell}^{\star}=0$ and, on noting (3.13), $\left.M_{\varepsilon}^{h}\left(W^{n}\right)\right|_{e_{k, \ell}}=M_{\varepsilon}^{h}\left(W^{n}\right)\left(\underline{v}_{\ell}\right)$. Setting $Q_{e_{k, \ell}}^{n}=\beta_{k, \ell}^{\star}-\alpha_{k, \ell}^{\star}=$ $-\alpha_{k, \ell}^{\star}<0$, we obtain from (3.31) that

$$
\left.\left|Q_{e_{k, \ell}}^{n}\right|\left|e_{k, \ell}\right| M_{\varepsilon}^{h}\left(W^{n}\right)\right|_{e_{k, \ell}}+Q_{e_{k, \ell}}^{n}\left(W_{\ell}^{n}-W_{k}^{n}\right)=0 .
$$

Similarly, if $\beta_{k, \ell}^{\star}>0$ then $W_{k}^{n}-W_{\ell}^{n}=\left|e_{k, \ell}\right| M_{\varepsilon}^{h}\left(W^{n}\right)\left(\underline{v}_{k}\right)$, and hence $W_{k}^{n}>W_{\ell}^{n}, \alpha_{k, \ell}^{\star}=0$ and $\left.M_{\varepsilon}^{h}\left(W^{n}\right)\right|_{e_{k, \ell}}=M_{\varepsilon}^{h}\left(W^{n}\right)\left(\underline{v}_{k}\right)$. Once again we set $Q_{e_{k, \ell}}^{n}=\beta_{k, \ell}^{\star}-\alpha_{k, \ell}^{\star}=\beta_{k, \ell}^{\star}>0$, and obtain 
from (3.31) the same relation (3.32) again. If both Lagrange multipliers, $\alpha_{k, \ell}^{\star}$ and $\beta_{k, \ell}^{\star}$, related to the edge $e_{k, \ell} \in \mathcal{E}_{I}^{h}$ are zero we set $Q_{e_{k, \ell}}^{n}=\beta_{k, \ell}^{\star}-\alpha_{k, \ell}^{\star}=0$, so (3.32) holds. Therefore, on setting $Q_{e_{k, \ell}}^{n}=\beta_{k, \ell}^{\star}-\alpha_{k, \ell}^{\star}$ for all $e_{k, \ell} \in \mathcal{E}_{I}^{h}$, and $Q_{e_{k, \ell}}^{n}=0$ for all $e_{k, \ell} \in \mathcal{E}_{B}^{h}$, we obtain that (3.32) holds for all $e_{k, \ell} \in \mathcal{E}^{h}$; that is, the equation in (3.9) holds for all $e_{k, \ell} \in \mathcal{E}^{h}$.

We now prove that the inequality in (3.9) holds for all $e_{k, \ell} \in \mathcal{E}^{h}$. It follows from (3.13) and as $W^{n} \in K^{h}\left(W^{n}\right)$ that if $W_{\ell}^{n} \geqslant W_{k}^{n}$ then $\left.M_{\varepsilon}^{h}\left(W^{n}\right)\right|_{e_{k, \ell}}=M_{\varepsilon}^{h}\left(W^{n}\right)\left(\underline{v}_{\ell}\right) \geqslant\left(W_{\ell}^{n}-W_{k}^{n}\right) /\left|e_{k, \ell}\right|$, and so $\left.|\psi|\left|e_{k, \ell}\right| M_{\varepsilon}^{h}\left(W^{n}\right)\right|_{e_{k, \ell}}+\psi\left(W_{\ell}^{n}-W_{k}^{n}\right) \geqslant(|\psi|+\psi)\left(W_{\ell}^{n}-W_{k}^{n}\right) \geqslant 0$ for all $\psi \in \mathbb{R}$. Similarly, if $W_{\ell}^{n} \leqslant W_{k}^{n}$ then $\left.M_{\varepsilon}^{h}\left(W^{n}\right)\right|_{e_{k, \ell}}=M_{\varepsilon}^{h}\left(W^{n}\right)\left(\underline{v}_{k}\right) \geqslant\left(W_{k}^{n}-W_{\ell}^{n}\right) /\left|e_{k, \ell}\right|$, and so $\left.|\psi|\left|e_{k, \ell}\right| M_{\varepsilon}^{h}\left(w^{n}\right)\right|_{e_{k, \ell}}+\psi\left(W_{\ell}^{n}-W_{k}^{n}\right) \geqslant(|\psi|-\psi)\left(W_{k}^{n}-W_{\ell}^{n}\right) \geqslant 0$ for all $\psi \in \mathbb{R}$. Hence (3.9), and so (3.8b), holds.

To show that (3.8a) holds we use the second inequality in (3.30), which yields that

$$
s_{j}\left(\frac{W_{j}^{n}-W_{j}^{n-1}}{\tau_{n}}-F_{j}^{n}\right)+\sum_{e_{k, j} \in \mathcal{E}_{I}^{h}}\left(\alpha_{k, j}^{\star}-\beta_{k, j}^{\star}\right)+\sum_{e_{j, \ell} \in \mathcal{E}_{I}^{h}}\left(\beta_{j, \ell}^{\star}-\alpha_{j, \ell}^{\star}\right)=0 \quad \forall \underline{v}_{j} \in \mathcal{V}_{I}^{h} ;
$$

and then note that $Q_{e_{k, \ell}}^{n}=\beta_{k, \ell}^{\star}-\alpha_{k, \ell}^{\star}$ for all $e_{k, l} \in \mathcal{E}_{I}^{h}$. Therefore $\left\{W^{n}, \underline{Q}^{n}\right\}_{n=2}^{N}$ satisfy (3.8a, b), and hence $(\mathrm{Q}),(3.4 \mathrm{a}, \mathrm{b})$.

As we have already proved existence of a solution $\left\{W^{n}\right\}_{n=2}^{N}$ to (P) for any $W^{1} \in K^{h}\left(W^{1}\right)$ such that $W^{1} \geqslant W^{0}$ in Theorem 3.2, the above establishes the existence, for the same initial condition $W^{1}$, of a solution $\left\{W^{n}, \underline{Q}^{n}\right\}_{n=2}^{N}$ to (Q).

If $\left\{W^{n}\right\}_{n=2}^{N}$ solves (P), an alternative way of finding $\left\{\underline{Q}^{n}\right\}_{n=2}^{N}$ such that $\left\{W^{n}, \underline{Q}^{n}\right\}_{n=2}^{N}$ solves $(\mathrm{Q})$ is given in the following lemma.

LEMMA 3.4 Let $\left\{W^{n}\right\}_{n=2}^{N}$ solve (P), (3.16) $\equiv$ (3.18). Let

$$
\underline{Y}^{h, n}:=\left\{\underline{\psi} \in \underline{V}^{h}:\langle\underline{\psi}, \underline{\nabla} \varphi\rangle=\left(\frac{W^{n}-W^{n-1}}{\tau_{n}}, \varphi\right)^{h}-\left(\widetilde{f}^{n}, \varphi\right) \quad \forall \varphi \in U_{0}^{h}\right\}, \quad n=2, \ldots, N .
$$

Then there exists $\underline{Q}^{n} \in \underline{Y}^{h, n}$ such that

$$
\left\langle\left|\underline{Q}^{n}\right|, M_{\varepsilon}^{h}\left(W^{n}\right)\right\rangle \leqslant\left\langle|\underline{\psi}|, M_{\varepsilon}^{h}\left(W^{n}\right)\right\rangle \quad \forall \underline{\psi} \in \underline{Y}^{h, n}, \quad n=2, \ldots, N .
$$

It follows that $\left\{W^{n}, \underline{Q}^{n}\right\}_{n=2}^{N}$ solves $(\mathrm{Q}),(3.4 \mathrm{a}, \mathrm{b}) \equiv(3.8 \mathrm{a}, \mathrm{b})$.

Proof. If $\left\{W^{n}\right\}_{n=2}^{N}$ solves (P), then we showed in Theorem 3.3 that there exists $\left\{\underline{Q}^{n}\right\}_{n=2}^{N}$ such that $\left\{W^{n}, \underline{Q}^{n}\right\}_{n=2}^{N}$ solves (Q), (3.4a, b). As $\underline{Q}^{n} \in \underline{Y}^{h, n}$, the affine manifold $\underline{Y}^{h, n}, n=2, \ldots, N$, is non-empty. Moreover, (3.4b) yields that $\underline{Q}^{n} \in \underline{Y}^{h, n}$ is such that

$$
\left\langle\left|\underline{Q}^{n}\right|, M_{\varepsilon}^{h}\left(W^{n}\right)\right\rangle+\left\langle\underline{Q}^{n}, \underline{\nabla} W^{n}\right\rangle \leqslant\left\langle|\underline{\psi}|, M_{\varepsilon}^{h}\left(W^{n}\right)\right\rangle+\left\langle\underline{\psi}, \underline{\nabla} W^{n}\right\rangle \quad \forall \underline{\psi} \in \underline{V}^{h}, \quad n=2, \ldots, N .
$$

As $\underline{\psi} \in \underline{Y}^{h, n}$ yields that

$$
\left\langle\underline{\psi}, \underline{\nabla} W^{n}\right\rangle=\left(\frac{W^{n}-W^{n-1}}{\tau_{n}}, W^{n}\right)^{h}-\left(\widetilde{f}^{n}, W^{n}\right), \quad n=2, \ldots, N,
$$


the desired result (3.34) follows immediately from (3.35).

Next, we prove the other half of the equivalence result in Theorem 3.3.

TheOREM 3.5 If $\left\{W^{n}, Q^{n}\right\}_{n=2}^{N}$ solves (Q), (3.4a,b) $\equiv(3.8 \mathrm{a}, \mathrm{b})$, then $\left\{W^{n}\right\}_{n=2}^{N}$ solves (P), (3.16) $\equiv(3.18)$.

Proof. Let $\left\{W^{n}, \underline{Q}^{n}\right\}_{n=2}^{N}$ solve (Q), (3.8a,b). Suppose that $\partial_{\downarrow}^{h} W^{n}\left(\underline{v}_{k}\right)>M_{\varepsilon}^{h}\left(W^{n}\right)\left(\underline{v}_{k}\right)$ at a vertex $\underline{v}_{k} \in \mathcal{V}^{h}$. Then there exists an edge $e \in \mathcal{E}^{h}, e=e_{k, j}$ or $e=e_{j, k}$, such that $W_{k}^{n}>W_{j}^{n}$ and $\left(W_{k}^{n}-W_{j}^{n}\right) /|e|>M_{\varepsilon}^{h}\left(W^{n}\right)\left(\underline{v}_{k}\right)=\left.M_{\varepsilon}^{h}\left(W^{n}\right)\right|_{e}$, on noting (3.13). However, then the inequality (3.9), and hence (3.8b), cannot be true. Therefore, it follows that $\partial_{\downarrow}^{h} W^{n}\left(\underline{v}_{k}\right) \leqslant M_{\varepsilon}^{h}\left(W^{n}\right)\left(\underline{v}_{k}\right)$ at every vertex $\underline{v}_{k} \in \mathcal{V}^{h}$, and so $W^{n} \in K^{h}\left(W^{n}\right)$.

It follows from (3.8a) that for any $\varphi \in K^{h}\left(W^{n}\right)$

$$
\begin{aligned}
\sum_{\underline{v}_{j} \in \mathcal{V}_{I}^{h}} s_{j}\left(\frac{W_{j}^{n}-W_{j}^{n-1}}{\tau_{n}}-F_{j}^{n}\right)\left(\varphi_{j}-W_{j}^{n}\right) & \\
& =\sum_{\underline{v}_{j} \in \mathcal{V}_{I}^{h}}\left(\sum_{e_{k, j} \in \mathcal{E}_{I}^{h}} Q_{e_{k, j}}^{n}-\sum_{e_{j, k} \in \mathcal{E}_{I}^{h}} Q_{e_{j, k}}^{n}\right)\left(\varphi_{j}-W_{j}^{n}\right)=: S .
\end{aligned}
$$

Since $\varphi=W^{n}=0$ at the boundary vertices, one can assume each edge $e \in \mathcal{E}_{I}^{h}$ appears twice on the right-hand side of (3.36) and, using (3.9), we obtain that

$$
\begin{aligned}
S & =\sum_{e_{k, j} \in \mathcal{E}_{I}^{h}} Q_{e_{k, j}}^{n}\left[\left(W_{k}^{n}-W_{j}^{n}\right)-\left(\varphi_{k}-\varphi_{j}\right)\right] \\
& =\sum_{e_{k, j} \in \mathcal{E}_{I}^{h}}\left[\left.\left|Q_{e_{k, j}}^{n}\right|\left|e_{k, j}\right| M_{\varepsilon}^{h}\left(W^{n}\right)\right|_{e_{k, j}}-Q_{e_{k, j}}^{n}\left(\varphi_{k}-\varphi_{j}\right)\right] .
\end{aligned}
$$

The latter sum is nonnegative. Indeed, $\partial_{\downarrow}^{h} \varphi\left(\underline{v}_{\ell}\right) \leqslant M_{\varepsilon}^{h}\left(W^{n}\right)\left(\underline{v}_{\ell}\right)$ for all $\underline{v}_{\ell} \in \mathcal{V}^{h}$. Hence, if $W_{k}^{n} \geqslant W_{j}^{n}$ then the equation in (3.9) yields that $Q_{e_{k, j}}^{n} \geqslant 0$, and (3.13) that $\left(\varphi_{k}-\varphi_{j}\right) /\left|e_{k, j}\right| \leqslant$ $M_{\varepsilon}^{h}\left(W^{n}\right)\left(\underline{v}_{k}\right)=\left.M_{\varepsilon}^{h}\left(W^{n}\right)\right|_{e_{k, j}}$. Similarly, if $W_{k}^{n}<W_{j}^{n}$ then $Q_{e_{k, j}}^{n} \leqslant 0$ and $\left(\varphi_{j}-\varphi_{k}\right) /\left|e_{k, j}\right| \leqslant$ $M_{\varepsilon}^{h}\left(W^{n}\right)\left(\underline{v}_{j}\right)=\left.M_{\varepsilon}^{h}\left(W^{n}\right)\right|_{e_{k, j}}$. This proves that $S \geqslant 0$, and hence, on recalling (3.36), that $W^{n}$ solves (3.18). Therefore $\left\{W^{n}\right\}_{n=2}^{N}$ solves (P).

\section{Steady state problem}

THEOREM 4.1 Let $\left\{W^{n}\right\}_{n=2}^{\infty}$ be a solution to (P) for $W^{1} \in K^{h}\left(W^{1}\right)$ with $W^{1} \geqslant W^{0}$ and $\widetilde{f}^{n} \geqslant 0$ for all $n \geqslant 2$. Then there exists $W \in U_{0}^{h}$ such that

$$
\lim _{n \rightarrow \infty} W^{n}=W \in K^{h}(W) \quad \text { and } \quad W^{0} \leqslant W^{1} \leqslant W^{n-1} \leqslant W^{n} \leqslant W \quad \forall n \geqslant 2 .
$$

In addition, if $\lim _{n \rightarrow \infty} \widetilde{f}^{n}=\widetilde{f}$ and $\lim _{n \rightarrow \infty} \tau_{n}=\tau>0$ then $W$ is a solution to the problem 
(P) Find $W \in K^{h}(W)$ such that

$$
0 \geqslant(\widetilde{f}, \varphi-W) \quad \forall \varphi \in K^{h}(W) .
$$

Proof. By Theorem 3.2, $W^{n-1} \leqslant W^{n}$ for any $n \geqslant 2$. Since $W^{n} \in K^{h}\left(W^{n}\right)$, we have also that $W^{n} \leqslant \mathcal{D}^{h}$ on recalling (3.20a). Therefore the monotonic increasing sequence $\left\{W^{n}\right\}_{n \geqslant 2}$ is bounded above. Hence, there exists $W \in U_{0}^{h}$ such that

$$
\lim _{n \rightarrow \infty} W^{n}=W \quad \text { and } \quad W^{0} \leqslant W^{1} \leqslant W^{n-1} \leqslant W^{n} \leqslant W \quad \forall n \geqslant 2 .
$$

To show that $W \in K^{h}(W)$, we note that $M_{\varepsilon}^{h}$ is continuous and so

$$
\partial_{\downarrow}^{h} W\left(\underline{v}_{j}\right)=\lim _{n \rightarrow \infty} \partial_{\downarrow}^{h} W^{n}\left(\underline{v}_{j}\right) \leqslant \lim _{n \rightarrow \infty} M_{\varepsilon}^{h}\left(W^{n}\right)\left(\underline{v}_{j}\right)=M_{\varepsilon}^{h}(W)\left(\underline{v}_{j}\right) \quad \forall \underline{v}_{j} \in \mathcal{V}^{h} .
$$

Furthermore, since $W \geqslant W^{n}$, for any $n \geqslant 2$, it follows from (3.14) and (3.17) that $K^{h}(W) \subset$ $K^{h}\left(W^{n}\right)$. Let $\varphi \in K^{h}(W)$, so that $\varphi \in K^{h}\left(W^{n}\right)$ for all $n \geqslant 2$. If $\widetilde{f}^{n} \rightarrow \widetilde{f}$ and $\tau_{n} \rightarrow \tau>0$ as $n \rightarrow \infty$, then passing to the limit $n \rightarrow \infty$ in (3.16) yields the desired inequality (4.1).

Associated with the steady state QVI problem $\left(\mathrm{P}_{S}\right)$ is the following stationary version of problem (Q):

$\left(Q_{S}\right)$ Find $W \in U_{0}^{h}$ and $\underline{Q} \in \underline{V}^{h}$ such that

$$
\begin{array}{rlrl}
-\langle\underline{Q}, \underline{\nabla} \varphi\rangle & =(\widetilde{f}, \varphi) & & \forall \varphi \in U_{0}^{h}, \\
\left\langle|\underline{\psi}|-|\underline{Q}|, M_{\varepsilon}^{h}(W)\right\rangle+\langle\underline{\psi}-\underline{Q}, \underline{\nabla} W\rangle \geqslant 0 & & \forall \underline{\psi} \in \underline{V}^{h} .
\end{array}
$$

We have the following analogues of Theorems 3.3 and 3.5, and Lemma 3.4.

Theorem 4.2 If $W$ solves $\left(\mathrm{P}_{S}\right),(4.1)$, then there exists $\underline{Q}$ such that $\{W, \underline{Q}\}$ solves $\left(\mathrm{Q}_{S}\right)$. Hence there exists a solution $\{W, \underline{Q}\}$ to $\left(\mathrm{Q}_{s}\right),(4.2 \mathrm{a}, \mathrm{b})$.

Proof. The proof is the direct analogue of that for the evolution case given in the proof of Theorem 3.3.

LEMMA 4.3 Let $W$ solve $\left(\mathrm{P}_{S}\right),(4.1)$. Let

$$
\underline{Y}^{h}:=\left\{\underline{\psi} \in \underline{V}^{h}:\langle\underline{\psi}, \underline{\nabla} \varphi\rangle=-(\widetilde{f}, \varphi) \quad \forall \varphi \in U_{0}^{h}\right\} .
$$

Then there exists $\underline{Q} \in \underline{Y}^{h}$ such that

$$
\left\langle|\underline{Q}|, M_{\varepsilon}^{h}(W)\right\rangle \leqslant\left\langle|\underline{\psi}|, M_{\varepsilon}^{h}(W)\right\rangle \quad \forall \underline{\psi} \in \underline{Y}^{h} .
$$

It follows that $\{W, \underline{Q}\}$ solves $\left(\mathrm{Q}_{s}\right),(4.2 \mathrm{a}, \mathrm{b})$.

Proof. The proof is the direct analogue of that for the evolution case given in the proof of Lemma 3.4.

THEOREM 4.4 If $\{W, \underline{Q}\}$ solves $\left(\mathrm{Q}_{S}\right),(4.2 \mathrm{a}, \mathrm{b})$, then $W$ solves $\left(\mathrm{P}_{S}\right),(4.1)$. 
Proof. The proof is the direct analogue of that for the evolution case given in the proof of Theorem 3.5.

In general, with different sources $\widetilde{f}^{n}, n \geqslant 2$, and starting from different initial states $W^{1}$ in Theorem 4.1, one arrives at different solutions to the steady state problem $\left(\mathrm{P}_{S}\right)$. However, we show below that if the source is strictly positive then the stationary solution $W$ is unique.

THEOREM 4.5 If $\widetilde{f} \geq 0$, then $\left(\mathrm{P}_{S}\right),(4.1)$, has a unique solution $W$. This solution is the same for every positive source $\widetilde{f}$ and satisfies

$$
W \geqslant W^{0}, \quad \partial_{\downarrow}^{h} W\left(\underline{v}_{k}\right) \geqslant k_{0} \quad \forall \underline{v}_{k} \in \mathcal{V}_{I}^{h} \quad \text { and } \quad W \geqslant \eta \quad \forall \eta \in K^{h}(\eta) .
$$

In addition, $W$ is the maximal steady state solution to $\left(\mathrm{P}_{S}\right)$; that is, if $W^{\prime}$ is any solution to $\left(\mathrm{P}_{S}\right)$ with a source $\widetilde{f}^{\prime} \geqslant 0$, then $W \geqslant W^{\prime}$ is also a solution to this problem. Furthermore, $W_{j}=W_{j}^{\prime}$ for every $\underline{v}_{j} \in \mathcal{V}_{I}^{h}$ such that $\int_{\Omega^{h}} \widetilde{f}^{\prime} \chi_{j} \mathrm{~d} \underline{x}>0$.

Proof. Existence of a solution $W \geqslant W^{0}$ to $\left(\mathrm{P}_{S}\right)$ follows from Theorem 4.1. If there exist two solutions $W^{(i)} \in K^{h}\left(W^{(i)}\right), i=1,2$, let $W^{\star} \in U_{0}^{h}$ be such that $W_{k}^{\star}=\max \left\{W_{k}^{(1)}, W_{k}^{(2)}\right\}$ for all $\underline{v}_{k} \in \mathcal{V}^{h}$. It follows from (3.21) that $W^{\star} \in K^{h}\left(W^{(i)}\right), i=1,2$, and, as $\widetilde{f}>0,\left(\widetilde{f}, W^{\star}-W^{(j)}\right)>0$ for either $j=1$ or 2 , which contradicts $W^{(j)}$ solving $\left(\mathrm{P}_{S}\right),(4.1)$. Therefore, the solution $W \in$ $K^{h}(W)$ to $\left(\mathrm{P}_{S}\right)$ is unique.

We now show that this unique solution is independent of the particular choice of $\widetilde{f}>0$. For $i=1,2$, let $W^{(i)} \in K^{h}\left(W^{(i)}\right)$ be the unique solution of $\left(\mathrm{P}_{S}\right),(4.1)$, with $\widetilde{f}=\widetilde{f}^{(i)}$. If $W^{(1)} \neq$ $W^{(2)}$, on defining $W^{\star} \in U_{0}^{h}$ as above it follows that $W^{\star} \in K^{h}\left(W^{(i)}\right), i=1,2$, and, as $\widetilde{f}^{(j)}>0$, $\left.\widetilde{(f}^{(j)}, W^{\star}-W^{(j)}\right)>0$ for either $j=1$ or 2 which contradicts $W^{(j)}$ solving $\left(\mathrm{P}_{S}\right)$, (4.1), with $\widetilde{f}=\widetilde{f}^{(j)}$. Therefore, $W^{(1)}=W^{(2)}$ and so the unique solution $W$ to $\left(\mathrm{P}_{S}\right)$ is independent of the particular choice of $\widetilde{f}>0$.

We now show that $\partial_{\downarrow}^{h} W\left(\underline{v}_{k}\right) \geqslant k_{0}$ for all $\underline{v}_{k} \in \mathcal{V}_{I}^{h}$. If not, let $\partial_{\downarrow}^{h} W\left(\underline{v}_{j}\right)<k_{0}$ for some $\underline{v}_{j} \in \mathcal{V}_{I}^{h}$ and then choose $W^{\star} \in U_{0}^{h}$ such that

$$
W_{k}^{\star}=W_{k}, \quad k \neq j, \quad \text { and } \quad W_{j}^{\star}>W_{j} \quad \text { with } \quad \partial_{\downarrow}^{h} W^{\star}\left(\underline{v}_{j}\right)=k_{0} .
$$

It follows from (3.11) that

$$
\partial_{\downarrow}^{h} W^{\star}\left(\underline{v}_{k}\right) \leqslant \partial_{\downarrow}^{h} W\left(\underline{v}_{k}\right) \leqslant M_{\varepsilon}^{h}(W)\left(\underline{v}_{k}\right), \quad k \neq j, \quad \text { and } \quad \partial_{\downarrow}^{h} W^{\star}\left(\underline{v}_{j}\right)=k_{0} \leqslant M_{\varepsilon}^{h}(W)\left(\underline{v}_{j}\right) .
$$

Hence, $W^{\star} \in K^{h}(W)$, and, as $\widetilde{f}>0,\left(\widetilde{f}, W^{\star}-W\right)>0$, which contradicts $W$ solving $\left(\mathrm{P}_{S}\right),(4.1)$. Therefore, the unique solution $W \in K^{h}(W)$ to $\left(\mathrm{P}_{S}\right)$ is such that $\partial_{\downarrow}^{h} W\left(\underline{v}_{k}\right) \geqslant k_{0}$ for all $\underline{v}_{k} \in \mathcal{V}_{I}^{h}$.

If $\eta \in K^{h}(\eta)$ and $W \neq \eta$, let $\eta^{\star} \in U_{0}^{h}$ be such that $\eta_{k}^{\star}=\max \left\{W_{k}, \eta_{k}\right\}$ for all $\underline{v}_{k} \in \mathcal{V}^{h}$. It follows from (3.21) that $\eta^{\star} \in K^{h}(W)$, and, as $\widetilde{f}>0,\left(\widetilde{f}, \eta^{\star}-W\right)>0$, which contradicts $W$ solving $\left(\mathrm{P}_{S}\right)$. Therefore, we have that $W \geqslant \eta$.

Finally, if $W^{\prime}$ solves $\left(\mathrm{P}_{S}\right)$ with a source $\tilde{f}^{\prime} \geqslant 0$ then (4.5) yields that $W \geqslant W^{\prime}$, and so $\left(\widetilde{f^{\prime}}, W-\right.$ $\left.W^{\prime}\right) \geqslant 0$. It follows from (3.14) and (3.17) that $W \in K^{h}(W) \subseteq K^{h}\left(W^{\prime}\right)$. Since $W^{\prime}$ is a solution to $\left(\mathrm{P}_{S}\right)$ with $\widetilde{f^{\prime}}$, only equality $\left(\widetilde{f^{\prime}}, W-W^{\prime}\right)=0$ is possible. In addition, $W$ is also a solution to $\left(\mathrm{P}_{S}\right)$ with $\widetilde{f}^{\prime}$. Moreover, $W=W^{\prime}$ in the "support" of $\widetilde{f}^{\prime}$ or, more precisely, $W_{j}=W_{j}^{\prime}$ for every $\underline{v}_{j} \in \mathcal{V}_{I}^{h}$ such that $\int_{\Omega^{h}} \widetilde{f}^{\prime} \chi_{j} d \underline{x}>0$. 


\section{Numerical solution}

\subsection{The augmented Lagrangian method}

In order to find a solution $\left\{W^{n}, \underline{Q}^{n}\right\}_{n=2}^{N}$ to (Q), (3.4a, b), we first find a solution $\left\{W^{n}\right\}_{n=2}^{N}$ to (P), (3.16), recall Lemma 3.4. For $n=2, \ldots, N$, we also note that $W^{n} \in K^{h}\left(W^{n}\right)$ is the unique solution to (3.29). We propose to solve this iteratively. Setting $W^{n, 0}=W^{n-1}$, then for $m \geqslant 1$ find

$$
W^{n, m}:=\arg \min _{W \in K^{h}\left(W^{n, m-1}\right)} G^{n}(W)
$$

until the sequence $\left\{W^{n, m}\right\}_{m \in \mathbb{N}}$ converges up to a given tolerance. Then set $W^{n}=W^{n, m}$.

In view of (3.19), the minimization problem (5.1) can be written as

$$
\begin{aligned}
& \min _{W \in K^{h}\left(W^{n, m-1}\right)} G^{n}(W) \\
& \equiv \min _{W \in U_{0}^{h}}\left\{G^{n}(W): \frac{W_{k}-W_{\ell}}{\left|e_{k, \ell}\right|} \in\left[-M_{\ell}^{n, m-1}, M_{k}^{n, m-1}\right] \quad \forall e_{k, \ell} \in \mathcal{E}_{I}^{h}\right\} \\
& \equiv \min _{W \in U_{0}^{h}, p \in \mathcal{A}^{h}}\left\{G^{n}(W)+\sum_{e_{k, \ell} \in \mathcal{E}_{I}^{h}} I_{\left[-M_{\ell}^{n, m-1}, M_{k}^{n, m-1}\right]}\left(p_{k, \ell}\right): \frac{W_{k}-W_{\ell}}{\left|e_{k, \ell}\right|}=p_{k, \ell} \quad \forall e_{k, \ell} \in \mathcal{E}_{I}^{h}\right\},
\end{aligned}
$$

where $M_{j}^{n, m-1}=M_{\varepsilon}^{h}\left(W^{n, m-1}\right)\left(\underline{v}_{j}\right)$ for all $\underline{v}_{j} \in \mathcal{V}^{h}$, and for $[a, b] \subset \mathbb{R}$,

$$
I_{[a, b]}(\zeta):=\left\{\begin{array}{cc}
0 & \zeta \in[a, b] \\
\infty & \zeta \notin[a, b]
\end{array}\right.
$$

is its indicator function.

We now extend the augmented Lagrangian method with splitting, see algorithm ALG2 on p. 170 in [14]. For all $W \in U_{0}^{h}$ and $p, \mu \in \mathcal{A}^{h}$, let

$$
\begin{aligned}
\mathcal{L}_{\rho}^{n, m-1}(W, p, \mu) & := \\
G^{n}(W)+\sum_{e_{k, \ell} \in \mathcal{E}_{I}^{h}}\left[I_{\left[-M_{\ell}^{n, m-1}, M_{k}^{n, m-1}\right]}\left(p_{k, \ell}\right)+\mu_{k, \ell}\left(\frac{W_{k}-W_{\ell}}{\left|e_{k, \ell}\right|}-p_{k, \ell}\right)\right. & \left.+\frac{\rho}{2}\left(\frac{W_{k}-W_{\ell}}{\left|e_{k, \ell}\right|}-p_{k, \ell}\right)^{2}\right]
\end{aligned}
$$

be the augmented Lagrangian, where $\rho \in \mathbb{R}_{>0}$ is a parameter. The splitting method is then:

Given $W^{n, 0} \in U_{0}^{h}$ and $p^{n, 0}, \mu^{n, 0} \in \mathcal{A}^{h}$, for $m \geqslant 1$

$$
\begin{aligned}
W^{n, m} & :=\arg \min _{W \in U_{0}^{h}} \mathcal{L}_{\rho}^{n, m-1}\left(W, p^{n, m-1}, \mu^{n, m-1}\right), \\
p^{n, m} & :=\arg \min _{p \in \mathcal{A}^{h}} \mathcal{L}_{\rho}^{n, m}\left(W^{n, m}, p, \mu^{n, m-1}\right), \\
\mu_{k, \ell}^{n, m} & =\mu_{k, \ell}^{n, m-1}+\rho\left(\frac{W_{k}^{n, m}-W_{\ell}^{n, m}}{\left|e_{k, \ell}\right|}-p_{k, \ell}^{n, m}\right) \quad \forall e_{k, \ell} \in \mathcal{E}_{I}^{h} .
\end{aligned}
$$


The minimization problem (5.5a) leads to the following well-posed linear system for $W^{n, m} \in U_{0}^{h}$

$$
\begin{aligned}
& s_{j} \frac{W_{j}^{n, m}-W_{j}^{n-1}}{\tau_{n}}+\sum_{e_{j, k} \in \mathcal{E}_{I}^{h}}\left[\frac{\mu_{j, k}^{n, m-1}}{\left|e_{j, k}\right|}+\frac{\rho}{\left|e_{j, k}\right|}\left(\frac{W_{j}^{n, m}-W_{k}^{n, m}}{\left|e_{j, k}\right|}-p_{j, k}^{n, m-1}\right)\right] \\
&-\sum_{e_{k, j} \in \mathcal{E}_{I}^{h}}\left[\frac{\mu_{k, j}^{n, m-1}}{\left|e_{k, j}\right|}+\frac{\rho}{\left|e_{k, j}\right|}\left(\frac{W_{k}^{n, m}-W_{j}^{n, m}}{\left|e_{k, j}\right|}-p_{k, j}^{n, m-1}\right)\right]=s_{j} F_{j}^{n}, \\
& \forall \underline{v}_{j} \in \mathcal{V}_{I}^{h} .
\end{aligned}
$$

The unique solution of $(5.5 \mathrm{~b})$ is

$$
p_{k, \ell}^{n, m}=\max \left\{-M_{\ell}^{n, m}, \min \left\{M_{k}^{n, m}, \frac{\mu_{k, \ell}^{n, m-1}}{\rho}+\frac{W_{k}^{n, m}-W_{\ell}^{n, m}}{\left|e_{k, \ell}\right|}\right\}\right\} \quad \forall e_{k, \ell} \in \mathcal{E}_{I}^{h} .
$$

The above is an extension of algorithm ALG2 in [14] from the variational inequality case to the QVI case. In the variational inequality case, e.g., $M_{\varepsilon}^{h}(\varphi) \equiv k_{0}>0$ or more generally a given positive $\varkappa \in C\left(\Omega^{h}\right)$, then $M_{j}^{n, m} \equiv M_{j} \equiv \varkappa\left(\underline{v}_{j}\right)$ for all $\underline{v}_{j} \in \mathcal{V}^{h}$ and the algorithm (5.5a-c) is guaranteed to converge for any choice of positive $\rho$; that is,

$$
W^{n, m} \rightarrow W^{n} \in U_{0}^{h}, \quad p^{n, m} \rightarrow p^{n} \in \mathcal{A}^{h}, \quad \mu^{n, m} \rightarrow \mu^{n} \in \mathcal{A}^{h} \quad \text { as } \quad m \rightarrow \infty,
$$

see [14]. Although we have no convergence proof of (5.5a-c) in the QVI case, in practice it was possible to obtain convergence even for reasonably small values of $\varepsilon$ in (3.12), see the numerical examples below, by adjusting the parameter $\rho$ and, if necessary, decreasing the time step $\tau_{n}$. We note that we have used a similar approach for the QVI problem arising in the modelling of growing sandpiles, see [5]. An alternative iterative numerical method for the QVI problem (Q), (3.4a, b), similar to that in [4], is based on the approximation of the non-differentiable nonlinearity $|\cdot|$ by $\frac{1}{r}|\cdot|^{r}$ with $0<r-1 \ll 1$.

If (5.5a-c) converges in the QVI case, i.e., (5.8) holds, then it follows from (5.5c) that

$$
p_{k, \ell}^{n}=\frac{W_{k}^{n}-W_{\ell}^{n}}{\left|e_{k, \ell}\right|} \quad \forall e_{k, \ell} \in \mathcal{E}_{I}^{h}
$$

On setting

$$
Q_{e_{k, \ell}}^{n}=\frac{\mu_{k, \ell}^{n}}{\left|e_{k, \ell}\right|} \quad \forall e_{k, \ell} \in \mathcal{E}_{I}^{h} \quad \text { and } \quad Q_{e_{k, \ell}}^{n}=0 \quad \forall e_{k, \ell} \in \mathcal{E}_{B}^{h}
$$

it follows from (5.8), (5.6) and (5.9) that (3.8a) holds. In addition, (5.8), (5.7), (5.9) and (5.10) yield for all $e_{k, \ell} \in \mathcal{E}_{I}^{h}$ that

$$
\begin{array}{lll}
p_{k, \ell}^{n}=M_{\varepsilon}^{h}\left(W^{n}\right)\left(\underline{v}_{k}\right) & \Rightarrow \mu_{k, \ell}^{n} \geqslant 0 & \Rightarrow Q_{e_{k, \ell}}^{n} \geqslant 0, \\
p_{k, \ell}^{n} \in\left(-M_{\varepsilon}^{h}\left(W^{n}\right)\left(\underline{v}_{\ell}\right), M_{\varepsilon}^{h}\left(W^{n}\right)\left(\underline{v}_{k}\right)\right) & \Rightarrow \quad \mu_{k, \ell}^{n}=0 & \Rightarrow Q_{e_{k, \ell}}^{n}=0, \\
p_{k, \ell}^{n}=-M_{\varepsilon}^{h}\left(W^{n}\right)\left(\underline{v}_{\ell}\right) & \Rightarrow \mu_{k, \ell}^{n} \leqslant 0 & \Rightarrow Q_{e_{k, \ell}}^{n} \leqslant 0 .
\end{array}
$$


Finally, it follows from (5.11), (5.9), (5.10) and (3.13) that (3.9), and hence (3.8b), holds. Therefore $\left\{W^{n}, \underline{Q}^{n}\right\}_{n=2}^{N}$ solve (Q), (3.8a,b).

Although the converged $\mu^{n} \in \mathcal{A}^{h}$ leads to $\underline{Q}^{n} \in \underline{V}^{h}$, via (5.10), so that $\left\{W^{n}, \underline{Q}^{n}\right\}_{n=2}^{N}$ solves $(\mathrm{Q})$, our numerical experiments showed that convergence, as $m \rightarrow \infty$, of $\mu^{n, m}$ is much slower than that of $W^{n, m}$. Even if $W^{n, m}$ converged with a severe tolerance, there still remained nodes from which the calculated flux $Q^{n, m}$ was not directed solely along the edge of steepest descent, but partly along some other edges too. Visually, this is exhibited in the unnatural river splits, which disappear from the river plots only after significantly more iterations. It was more efficient to find $Q^{n}$ using Lemma 3.4 as follows.

Given $\left\{W^{n}\right\}_{n=2}^{N}$ solving (P), (3.16) $\equiv(3.18)$, we find $\left\{\underline{Q}^{n}\right\}_{n=2}^{N}$ such that $\left\{W^{n}, \underline{Q}^{n}\right\}_{n=2}^{N}$ solves $(\mathrm{Q}),(3.4 \mathrm{a}, \mathrm{b}) \equiv(3.8 \mathrm{a}, \mathrm{b})$, by recasting the weighted $L^{1}$ minimization problem (3.34) as a standard linear programming problem. On recalling (3.1), we know for $n=2, \ldots, N$ that

$$
\underline{Q}^{n}=\sum_{e_{k, \ell} \in \mathcal{E}_{I}^{h}} Q_{e_{k, \ell}}^{n} \underline{i}_{e_{k, \ell}} \mathrm{d} \mathcal{H}^{1}\left(e_{k, \ell}\right),
$$

where $\left\{Q_{e_{k, \ell}}^{n}\right\}_{e_{k, \ell} \in \mathcal{E}_{I}^{h}}, Q_{e_{k, \ell}}^{n} \in \mathbb{R}$, are to be determined. As we know $W^{n}$, the signs of these fluxes are also known. It follows from (3.10(i),(iii)) that $Q_{e_{k, \ell}}^{n} \geqslant(\leqslant) 0$ if $W_{k}^{n}>(<) W_{\ell}^{n}$ and $Q_{e_{k, \ell}}^{n}=0$ if $W_{k}^{n}=W_{\ell}^{n}$. Therefore, for every $e_{k, \ell} \in \mathcal{E}_{I}^{h}$ we set

$$
\mathcal{S}_{k, \ell}^{n}=\left\{\begin{aligned}
1 & W_{k}^{n}>W_{\ell}^{n}, \\
-1 & W_{k}^{n} \leqslant W_{\ell}^{n}
\end{aligned}\right.
$$

so that $\widetilde{Q}_{k, \ell}^{n}:=\mathcal{S}_{k, \ell}^{n} Q_{e_{k, \ell}}^{n}=\left|Q_{e_{k, \ell}}^{n}\right|$. In this notation, the minimization problem (3.34) can be rewritten, on recalling (3.6), as find $\underline{\widetilde{Q}}^{n} \in \underline{\widetilde{Y}}^{h, n}$ such that

$$
\sum_{e_{k, \ell} \in \mathcal{E}_{I}^{h}} a_{k, \ell}^{n} \widetilde{Q}_{k, \ell}^{n} \leqslant \sum_{e_{k, \ell} \in \mathcal{E}_{I}^{h}} a_{k, \ell}^{n} \widetilde{Q}_{k, \ell} \quad \forall \underline{\widetilde{Q}} \in \underline{\widetilde{Y}}^{h, n}
$$

where $a_{k, \ell}^{n}=\left.\left|e_{k, \ell}\right| M_{\varepsilon}^{h}\left(W^{n}\right)\right|_{e_{k, \ell}}$ and

$$
\begin{aligned}
& \underline{\widetilde{Y}}^{h, n}:=\left\{\underline{\psi} \in \mathcal{A}_{\geqslant 0}^{h}: \sum_{e_{j, k} \in \mathcal{E}_{I}^{h}} \mathcal{S}_{j, k}^{n} \psi_{j, k}-\sum_{e_{k, j} \in \mathcal{E}_{I}^{h}} \mathcal{S}_{k, j}^{n} \psi_{k, j}=s_{j}\left(F_{j}^{n}-\frac{W_{j}^{n}-W_{j}^{n-1}}{\tau_{n}}\right)\right. \\
& \left.\forall \underline{v}_{j} \in \mathcal{V}_{I}^{h}\right\} .
\end{aligned}
$$

The minimization problem (5.13) is a linear programming problem, and can be solved efficiently using a standard procedure; e.g. linprog, see [19]. We note that a different method, applicable to more general $L^{1}$ optimization problems (see, e.g., [10, p. 8]), reduces (3.34) to a linear programming problem of higher dimension which, nevertheless, can also be solved very efficiently.

\section{$5.2 \quad$ Numerical simulations}

We precede the presentation of our numerical experiments by the following comment. Convergence, even in some weak sense, of solutions $\left\{W^{n}, \underline{Q}^{n}\right\}_{n=1}^{N}$ of (Q), (3.4a,b), as the mesh parameters 
$h, \tau \rightarrow 0$ is difficult to expect in general because of two reasons. First, as was noted above, the problem can be ill-conditioned in that small changes in the relief, $w_{0}$, can, in some cases, lead to dramatic changes of the river network. Second, limiting the possible flow direction to the direction of the mesh edges can, in principle, lead to a non-negligible distortion of the river network. Nevertheless, we expect a river (or wadi) with a pronounced valley to be well approximated by a nearby zigzag path consisting of the mesh edges. Traditional lattice D8-algorithm-based methods suffer from the same problems but, usually, are able to produce realistic hydrological maps. In the numerical simulations presented in this section we use artificial landscapes to illustrate some typical features of our approximation.

We start with a radially-symmetric non-regularized $\left(\varepsilon=0\right.$ and $\left.k_{0}=0\right)$ problem having an analytical solution. We chose the relief in the form of a cone surrounded by a moat, see the left of Figure 1. In polar coordinates $\{r, \theta\}$

$$
w_{0}(r)= \begin{cases}0.3-r & 0 \leqslant r \leqslant 0.6 \\ r-0.9 & 0.6 \leqslant r \leqslant 0.9 \\ 0 & r \geqslant 0.9\end{cases}
$$

Let $f=1$ for $r \leqslant 0.2$ and $f=0$ outside of this disc. Rain water flows down the cone slopes, so $q(\underline{x}, t)=q(|\underline{x}|, t) \underline{\hat{x}}$, and gradually fills the moat. Here $\underline{\hat{x}}$ is the unit vector in the direction $\underline{x}$. Equating the volumes of the discharged and the collected water, it is a simple matter to find the height, $H(t)$, of the water layer above the lowest circle, $r=0.6$, of the moat. This leads to $H(t)=\sqrt{t / 30}$ for $t<2.7$. The flux $q$ on the cone slopes above this layer, i.e. for $r<0.6-H(t)$, can be found from the mass balance equation $\partial_{r}(r q)=r f$ with the initial condition $q(0, t)=0$, yielding

$$
q(r, t)= \begin{cases}0.5 r & 0 \leqslant r \leqslant 0.2, \\ 0.02 r^{-1} & 0.2<r<0.6-H(t), \\ \text { undetermined } & 0.6-H(t)<r<0.6+H(t), \\ 0 & r \geqslant 0.6+H(t) .\end{cases}
$$

Numerical solutions have been obtained with $\varepsilon=0.01$ and $k_{0}=0.005$ (the model regularization parameters), $\Omega=(-1,1)^{2}, W^{1}=W^{0}, \tau_{n}=0.01$ (the constant time step) and $\rho=0.01$ (the augmented Lagrangian parameter), and compared to the analytical solution at $t=0.5$. For computational ease, the integral $\left(\widetilde{f}^{n}, \cdot\right)$ on the right-hand sides of (3.4a) and (3.16) was replaced by $\left(\widetilde{f}^{n}, \cdot\right)^{h}$, and hence similarly in the definition of $F_{j}^{n}$ in (3.7).

Comparing the approximate surface $W\left(\cdot, t_{n}\right) \equiv W^{n}(\cdot)$ and the exact one, $w\left(\cdot, t_{n}\right)$, is straightforward; we calculated the relative error in the approximate $L^{1}$ norm:

$$
\frac{\sum_{\sigma \in \mathcal{T}^{h}}|\sigma|\left|w\left(\underline{x}^{(\sigma)}, t_{n}\right)-W^{n}\left(\underline{x}^{(\sigma)}\right)\right|}{\sum_{\sigma \in \mathcal{T}^{h}}|\sigma|\left|w\left(\underline{x}^{(\sigma)}, t_{n}\right)\right|},
$$

where $\underline{x}^{(\sigma)}$ is the centroid of $\sigma$. The flux comparison is far more complicated, as it requires to compare the exact flow field $q\left(\cdot, t_{n}\right)$ (continuous in this example, but a measure in general) and the singular vectorial measure $\underline{Q}^{\bar{n}}(\cdot)$.

For a very crude check of the flux accuracy in this example we used the continuous radial vector fields $\underline{\phi}_{\zeta}(\underline{x})=|\underline{x}|^{\zeta-1} \underline{x}$, where $\zeta \in \mathbb{R}_{>0}$, for which the integrals $I_{\zeta}(t)=\int_{\Omega(t)} \underline{q}(\underline{x}, t) . \underline{\phi}_{\zeta}(\underline{x}) \mathrm{d} \underline{x}$ 
can be found analytically. Here $\Omega(t)$ is the disc centered at the origin with radius $R(t)=0.6-H(t)$ in which the exact flux is known. The integrals were compared, for several $\zeta$ values, to the following approximation of the duality pairing of $\underline{Q}^{n}$ and $\underline{\phi}_{\zeta}$ on $\Omega(t)$ :

$$
\sum_{\left|\underline{x}^{(e)}\right| \leqslant R\left(t_{n}\right)}|e|\left|Q^{n}\right|\left|\underline{x}^{(e)}\right|^{\zeta-1} \underline{i}_{e} \cdot \underline{x}^{(e)}
$$

where $\underline{x}^{(e)}$ is the midpoint of edge $e \in \mathcal{E}_{I}^{h}$.

We used two triangulations having approximately the same number of elements and not in any way fitted to the relief $w_{0}$ or the source $f$. The first mesh was a general Matlab-generated triangular mesh with the maximal element size $h=0.025$; and the second was a uniform mesh, which was obtained by first dividing $\Omega$ into squares with side length $2 / 100=0.02$ and then dividing each square into two triangles by its SW-NE diagonal. The surface $w(\cdot, 0.5)$ was found numerically (Figure 1, right) with the relative $L^{1}$ error not exceeding $0.2 \%$ for both meshes, recall (5.16). For the non-uniform mesh, the approximate edge fluxes $Q^{n}$ yield the runoff picture seen in the left of Figure 2. Here and below, to show the network of river channels we plot the edges $e \in \mathcal{E}_{I}^{h}$ for which the calculated flux $\left|Q_{\rho}^{n}\right|$ exceeds some threshold, with the plotted edges being thicker for a stronger flux, and the plotting of "rivers" in "lakes" being suppressed. We see that the continuous water flow, (5.15), is approximated by a set of channels bringing the discharged water towards the lake that forms in the moat around the cone. Although the position of the channels seem random, their azimuthal distribution is sufficiently uniform. For $\zeta=0.1,0.25,0.5$ and 1 , the integrals $I_{\zeta}(0.5)$ have been approximated by (5.17) with relative errors smaller than $0.5 \%$.

Although the errors in $I_{\zeta}(0.5)$ were only about double those for the non-uniform mesh, the calculated channels in this case are strongly influenced by the anisotropy of the mesh (Figure 2, right). This effect is especially strong, because the conical surface itself has no pronounced relief features such as typical river valleys of natural landscapes. D8 algorithms show a similar behavior [11]; another well-known consequence of using a uniform mesh is the abundance of parallel channels generated, especially, in flat areas [21].

For the "rippled" conical support

$$
w_{0}(r, \theta)= \begin{cases}\max \{-0.95+r, 0.85-r(1+0.25|\sin (5 \theta)|)\} & r \leqslant 0.95, \\ 0 & r>0.95,\end{cases}
$$

see Figure 3; the approximate drainage channels generally follow ten relief valleys, see Figure 4 for the simulation results computed at $t=0.06$, before the time when the ten separated lakes begin
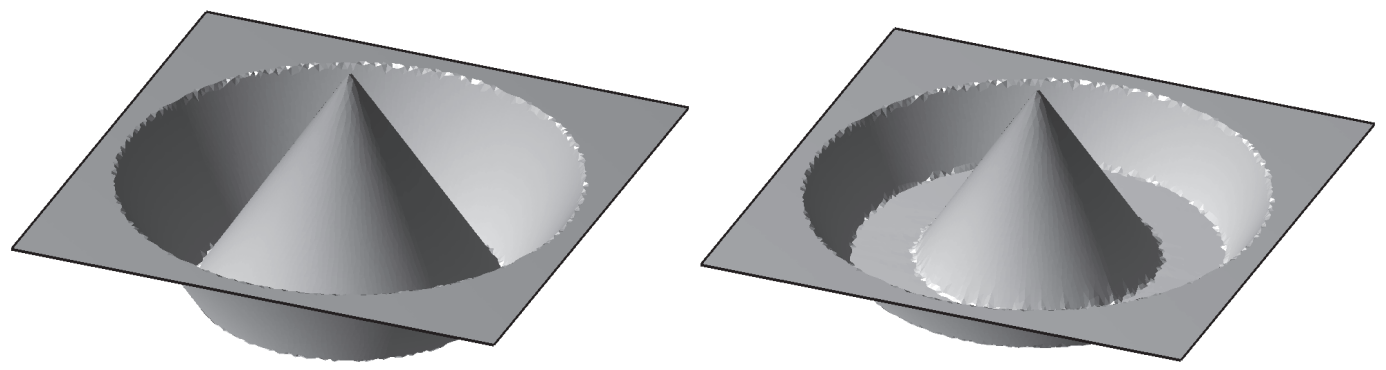

FIG. 1. Left: initial support $w_{0}$; right: the approximate surface $W(\cdot, 0.5)$ 

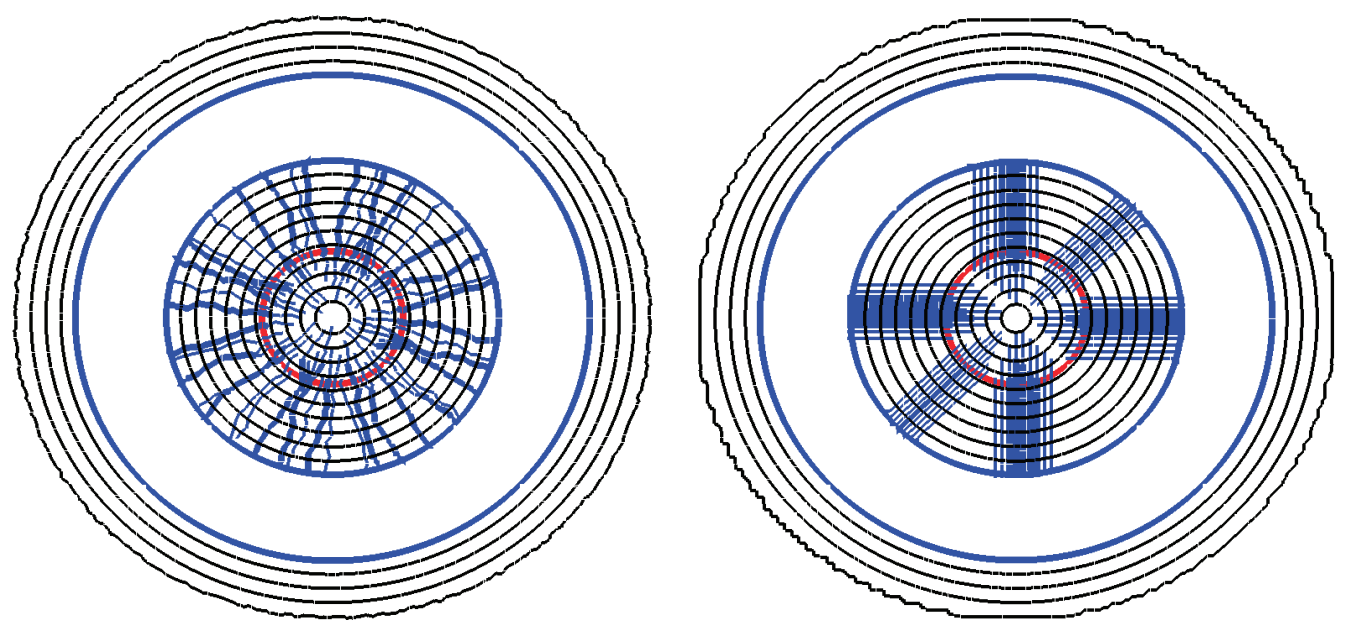

FIG. 2. Computed drainage channels and exact lake boundaries (blue lines) at $t_{n}=0.5$. Also shown: level contours of $W^{n}$ (black) and the source support boundary (red line). Left: results for a non-uniform mesh; right: results for the uniform mesh

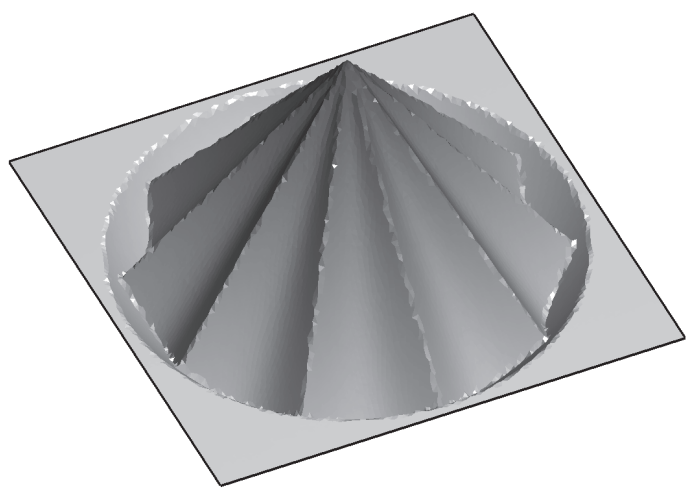

FIG. 3. Rippled support $w_{0}$

to merge, with the same non-uniform (left) and uniform (right) meshes as in the previous example. In addition, the numerical scheme parameters were the same as before, except here the time step $\tau_{n}=0.002$. Due to the presence of "river valleys", the influence of the anisotropy of the uniform mesh is weaker for this relief.

All simulations have been performed in Matlab R2012b (64 bit) on a PC with an Intel Core i5$24003.10 \mathrm{GHz}$ processor and $16 \mathrm{~GB}$ RAM. The primal QVI was solved using the extended ALG2 algorithm as described in Subsection 5.1 with the stopping criterion based solely on the convergence of $W^{n, m}$ : the iterations were stopped if the relative change of this variable in the approximate $L^{1}$ norm, the analogue of (5.16), is less than $10^{-7}$. In these examples, computing $W^{n}, n=2, \ldots, N$ (all time levels) took 2-3 minutes of CPU time. Then the approximate flux $\underline{Q}^{N}$ was computed (with 

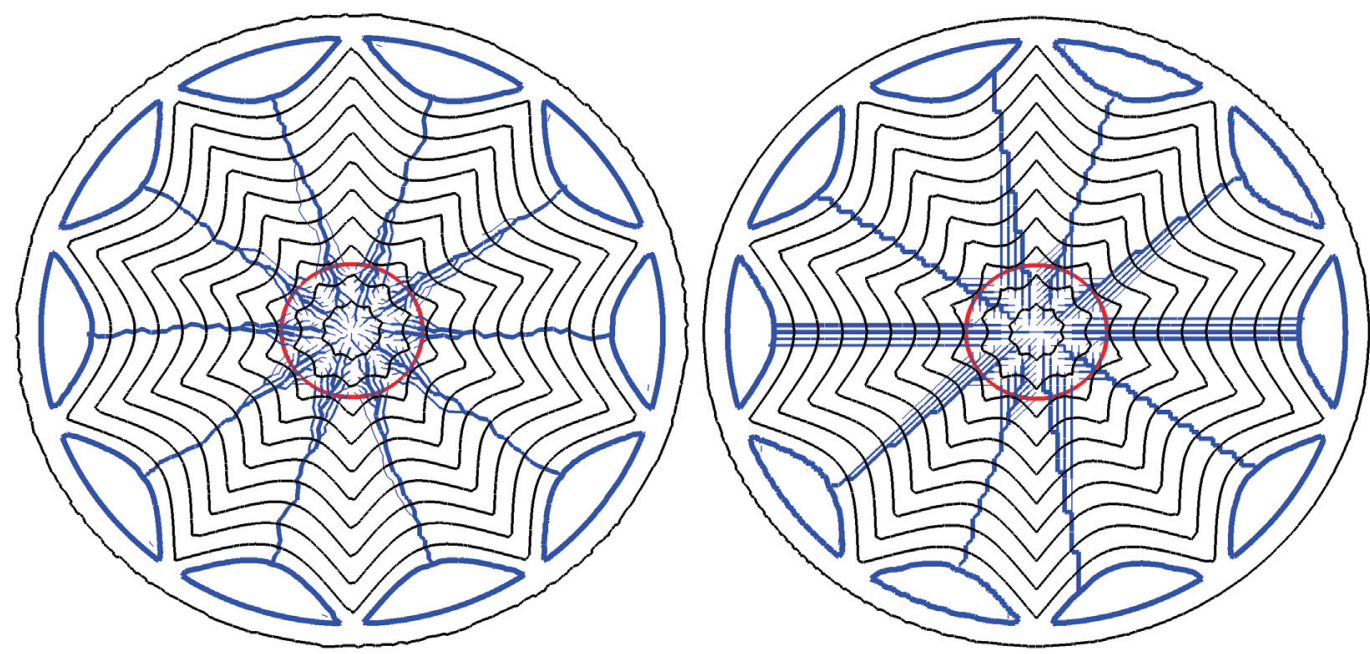

FIG. 4. Computed drainage channels at $t=0.06$ for the relief $w_{0}$ shown in Figure 3. Left: non-uniform mesh; right: uniform mesh

a similar tolerance) in a few seconds using the standard Matlab linear programming solver, linprog, via the rewritten formulation (5.13) of (3.34).

\section{Lattice model approach}

In DEMs it is difficult to distinguish the actual topographic depressions from false ones, caused by a forest canopy, a bridge, a missed narrow gorge of a river etc. To ensure drainage continuity, it is common practice to rectify all depressions and flat areas prior to any DEM usage in hydrogeomorphic applications. In principle, it is possible to fill all depressions in the initial relief $W^{0}$ by solving the regularized evolutionary problem $(\mathrm{P})$ until the steady state is reached. For $k_{0}>0$ the resulting surface $W$ is also free of the flat horizontal areas, recall Theorem 4.5. This approach is, however, too slow for practical applications that need high resolution DEMs of large areas. Typically, modern GIS work with massive data sets containing around $10^{6}$ to $10^{8}$ cell elevations, see, e.g., [20]; such DEMs are widely available. Solving problem (P) on a finite element mesh representing the Earth's relief with such accuracy is not practical.

Efficient drainage network extraction algorithms in GIS do not solve any evolutionary problems. Most of these lattice algorithms first replace the raster (square-grid) DEM, representing the relief, by a new one, where all pits (lakes) are filled. Then they treat the flat areas and determine the direction of flow out of each cell. Finally, the accumulated water flux through every cell is computed. We now present such algorithms in the context of our finite element model, which uses a TIN relief representation and directs water fluxes along the mesh edges. 


\subsection{Lake filling, flow routing and flux accumulation}

We now consider the unregularized case, $\varepsilon=0$, and define, similarly to (3.17) and (3.12), for any $\eta \in U_{0}^{h}$

$$
\begin{aligned}
K_{0}^{h}(\eta) & \left.:=\left\{\varphi \in U_{0}^{h}: \partial_{\downarrow}^{h} \varphi\left(\underline{v}_{j}\right) \leqslant M_{0}^{h}(\eta) \underline{v}_{j}\right) \quad \forall \underline{v}_{j} \in \mathcal{V}^{h}\right\}, \\
\text { where } \quad M_{0}^{h}(\eta)\left(\underline{v}_{j}\right) & = \begin{cases}k_{0} & \eta_{j}>W_{j}^{0}, \\
\max \left\{k_{0}, \partial_{\downarrow}^{h} W^{0}\left(\underline{v}_{j}\right)\right\} & \eta_{j} \leqslant W_{j}^{0} .\end{cases}
\end{aligned}
$$

It follows from (6.1a,b), (3.12) and (3.17) that for any $\varepsilon>0$ and any $\eta \in U_{0}^{h}$

$$
\left.M_{0}^{h}(\eta)\left(\underline{v}_{j}\right) \leqslant M_{\varepsilon}^{h}(\eta) \underline{v}_{j}\right) \quad \forall \underline{v}_{j} \in \mathcal{V}^{h} \quad \Rightarrow \quad K_{0}^{h}(\eta) \subseteq K^{h}(\eta) .
$$

For the given Earth relief $W^{0}$, we now construct a new relief, $\widetilde{W}^{0}$, such that

$$
W^{0} \leqslant \widetilde{W}^{0} \in K_{0}^{h}\left(\widetilde{W}^{0}\right) \subseteq K^{h}\left(\widetilde{W}^{0}\right) \quad \text { and } \quad \partial_{\downarrow}^{h} \widetilde{W}^{0}\left(\underline{v}_{j}\right) \geqslant k_{0} \quad \forall \underline{v}_{j} \in \mathcal{V}_{I}^{h}
$$

using the following iterative algorithm.

1. Set the "water level" $L_{j}=+\infty$ if $\underline{v}_{j} \in \mathcal{V}_{I}^{h}$ and $L_{j}=0$ if $\underline{v}_{j} \in \mathcal{V}_{B}^{h}$.

2. Set $f$ lag $=0$. For each $e(k, \ell) \in \mathcal{E}_{I}^{h}$ :

- Set

$$
\begin{aligned}
& L_{k}^{\prime}=\max \left\{W_{k}^{0}, \min \left\{L_{k}, L_{\ell}+k_{0}|e(k, \ell)|\right\}\right\}, \\
& L_{\ell}^{\prime}=\max \left\{W_{\ell}^{0}, \min \left\{L_{\ell}, L_{k}+k_{0}|e(k, \ell)|\right\}\right\} .
\end{aligned}
$$

- If $L_{k}^{\prime} \neq L_{k}$ or $L_{\ell}^{\prime} \neq L_{\ell}$, set flag $=1$.

- Set $L_{k}=L_{k}^{\prime}$ and $L_{\ell}=L_{\ell}^{\prime}$.

3. If flag $=1$ go to 2 .

4. Set $\widetilde{W}^{0}=L \in U_{0}^{h}$.

The second condition in (6.3) yields that we obtain a relief without depressions and flat areas. For $k_{0}>0$ very small, the relief is almost horizontal in the "lake" domain $\widetilde{W}^{0}>W^{0}$, as $\partial_{\downarrow}^{h} \widetilde{W}^{0}\left(\underline{v}_{j}\right)=$ $k_{0}$ if $\widetilde{W}^{0}\left(\underline{v}_{j}\right)>W^{0}\left(\underline{v}_{j}\right)$.

THEOREM 6.1 The above iterative algorithm yields $\widetilde{W}^{0} \in U_{0}^{h}$ satisfying (6.3). Moreover, there exists only one function satisfying (6.3). Furthermore, $\widetilde{W}^{0} \leqslant W$, where $W$ is the unique solution of $\left(\mathrm{P}_{S}\right)$ for any $\widetilde{f}>0$.

Proof. On the first iteration of all edges $e(k, \ell) \in \mathcal{E}_{I}^{h}, L_{j}$ values become finite at all $\underline{v}_{j} \in \mathcal{V}_{I}^{h}$ connected by an edge to a boundary node. Then at the next iteration those connected to these nodes become finite, and so on. Finally the $L_{j}$ values at every $\underline{v}_{j} \in \mathcal{V}_{I}^{h}$ become finite in a finite number of iterations. Obviously, throughout the iterations $L_{j}$ is monotonically decreasing and $L_{j} \geqslant W_{j}^{0}$ for all $\underline{v}_{j} \in \mathcal{V}_{I}^{h}$. Hence we have that $\widetilde{W}^{0} \geqslant W^{0}$.

Whenever the value of $L_{\ell}$ strictly decreases, either $L_{\ell}^{\prime}=L_{k}+k_{0}|e(k, \ell)|$ or $L_{\ell}^{\prime}=W_{\ell}^{0} \geqslant$ $\min \left\{L_{\ell}, L_{k}+k_{0}|e(k, \ell)|\right\}$ for some $e(k, \ell) \in \mathcal{E}_{I}^{h}$. Since $L_{k} \geqslant L_{k}^{\prime}$, in the first case we get $L_{\ell}^{\prime} \geqslant$ $L_{k}^{\prime}+k_{0}|e(k, \ell)|$ so there is an edge along which $L^{\prime}$ decreases from $\underline{v}_{\ell}$ with at least the slope $k_{0}$. 
In the second case, since $L_{\ell}>W_{\ell}^{0}$, we obtain that $L_{\ell}^{\prime} \geqslant L_{k}+k_{0}|e(k, \ell)| \geqslant L_{k}^{\prime}+k_{0}|e(k, \ell)|$. Therefore for any $\underline{v}_{\ell} \in \mathcal{V}_{I}^{h}$ after a strict decrease of $L_{\ell}$, we have that $\partial_{\downarrow}^{h} L\left(\underline{v}_{\ell}\right) \geqslant k_{0}$.

If at an iteration the level at $\underline{v}_{\ell}$ remains unchanged, $L_{\ell}^{\prime}=L_{\ell}$, edge descents of $L^{\prime}$ from $\underline{v}_{\ell}$ can only become steeper since the $L^{\prime}$ levels at the neighboring nodes do not increase. As $\partial_{\downarrow}^{h} \widetilde{L}\left(\underline{v}_{\ell}\right) \geqslant k_{0}$ from a previous strict decrease in $L_{\ell}$, e.g. becoming finite, we have that this remains true. Hence, we have that $\partial_{\downarrow}^{h} \widetilde{W}^{0}\left(\underline{v}_{j}\right) \geqslant k_{0}$ at every $\underline{v}_{j} \in \mathcal{V}_{I}^{h}$.

To show that $\widetilde{W}^{0} \in K_{0}^{h}\left(\widetilde{W}^{0}\right)$, we note that if $\widetilde{W}_{\ell}^{0}=W_{\ell}^{0}$ then $\widetilde{W}_{\ell}^{0}-\widetilde{W}_{k}^{0} \leqslant W_{\ell}^{0}-W_{k}^{0}$ for all $e(k, \ell) \in \mathcal{E}^{h}$. Hence $\partial_{\downarrow}^{h} \widetilde{W}^{0}\left(\underline{v}_{\ell}\right) \leqslant \partial_{\downarrow}^{h} W^{0}\left(\underline{v}_{\ell}\right) \leqslant M_{0}^{h}\left(W^{0}\right)\left(\underline{v}_{\ell}\right)$, on recalling (6.1b). Otherwise, if $\widetilde{W}^{0}\left(\underline{v}_{\ell}\right)>W^{0}\left(\underline{v}_{\ell}\right)$, we have that $\widetilde{W}^{0}\left(\underline{v}_{\ell}\right) \leqslant \widetilde{W}^{0}\left(v_{k}\right)+k_{0}|e(k, \ell)|$ for all $e(k, \ell) \in \mathcal{E}^{h}$, so that $\partial_{\downarrow}^{h} \widetilde{W}^{0}\left(\underline{v}_{\ell}\right) \leqslant k_{0}=M_{0}^{h}\left(\widetilde{W}^{0}\right)\left(\underline{v}_{\ell}\right)$ also in this case. Hence, on recalling (6.1 a) and (6.2), it follows that $\widetilde{W}^{0} \in K_{0}^{h}\left(\widetilde{W}^{0}\right) \subseteq K^{h}\left(\widetilde{W}^{0}\right)$.

Suppose that $W^{(1)}$ and $W^{(2)}$ satisfy (6.3) and $W_{\ell}^{(1)}<W_{\ell}^{(2)}$ at some vertex $\underline{v}_{\ell} \in \mathcal{V}_{I}^{h}$. Choose an edge path from $\underline{v}_{\ell}$ to the boundary $\partial \Omega^{h}$ such that at each vertex the outflow edge is the steepest descent of $W^{(1)}$. Let $\underline{v}_{k}$ be the vertex associated with edge $e(k, \ell)$ of this path. Then $\left[W_{\ell}^{(2)}-\right.$ $\left.W_{k}^{(2)}\right] /|e(k, \ell)| \leqslant \partial_{\downarrow}^{h} W^{(2)}\left(\underline{v}_{\ell}\right) \leqslant k_{0}$, since $W_{\ell}^{(2)}>W_{\ell}^{(1)} \geqslant W_{\ell}^{0}$ and $W^{(2)} \in K_{0}^{h}\left(W^{(2)}\right)$. On the other hand, at $\underline{v}_{\ell}$ the edge $e(k, \ell)$ is the steepest descent edge for $W^{(1)}$. From (6.3) it follows that $\left[W_{\ell}^{(1)}-W_{k}^{(1)}\right] /|e(k, \ell)|=\partial_{\downarrow}^{h} W^{(1)}\left(\underline{v}_{\ell}\right) \geqslant k_{0}$. Hence $W^{(1)}$ decreases along the edge $e(k, \ell)$ at least as fast as $W^{(2)}$, and so the inequality $W^{(1)}<W^{(2)}$ holds also at the next vertex, $\underline{v}_{k}$, of the path. Continuing, we arrive at a contradiction at the last vertex since both functions must be zero at the boundary nodes.

Finally, it immediately follows from $\widetilde{W}^{0} \in K^{h}\left(\widetilde{W}^{0}\right)$ and (4.5) that $\widetilde{W}^{0} \leqslant W$.

Although, the conditions (6.3) satisfied uniquely by $\widetilde{W}^{0}$ are very similar to those, (4.5), satisfied by $W$, the unique solution of $\left(\mathrm{P}_{S}\right)$ for any $\widetilde{f}>0$, we only know that $W \geqslant \widetilde{W}^{0}$. The following simple example shows that $W$ may not be $\widetilde{W}^{0}$.

Let $\Omega^{h} \equiv[0,4]$ with nodes $\underline{v}_{j}=j, j=0, \ldots, 4$, and $W_{1}^{0}=W_{3}^{0}=k_{0}$ and $W_{2}^{0}=-2 k_{0}$. It follows that $\widetilde{W}_{1}^{0}=\widetilde{W}_{3}^{0}=k_{0}$ and $\widetilde{W}_{2}^{0}=2 k_{0}$. Now consider $W^{1} \equiv \widetilde{W}^{0}$ for the evolutionary problem (P), (3.16), with $f^{n} \equiv \widetilde{f}>0$ for all $n \geqslant 2$. We see that $W^{1}$ is not the steady state solution $W$ of $\left(\mathrm{P}_{S}\right)$, since "water" coming into node $\underline{v}_{1}$ from the source cannot flow out as $W_{1}^{1}=W_{1}^{0}$ and so $M_{\varepsilon}^{h}\left(W^{1}\right)\left(\underline{v}_{1}\right)=\partial_{\downarrow}^{h} W^{0}\left(\underline{v}_{1}\right)=3 k_{0}>\bar{k}_{0}=\partial_{\downarrow}^{h} W^{1}\left(\underline{v}_{1}\right)$. Therefore $W^{1}$ increases. A simple calculation yields that the steady state solution $W$ is such that $W_{1}=W_{3}=k_{0}+a$ and $W_{2}=$ $2 k_{0}+a$, where $a=2 k_{0} \varepsilon /\left(2 k_{0}+\varepsilon\right)$ for any $\varepsilon>0$.

Having constructed a new relief $\widetilde{W}^{0}$ via the above iterative algorithm, we now consider the flow routing. This is now trivial as $\partial_{\downarrow}^{h} \widetilde{W}^{0}\left(\underline{v}_{k}\right) \geqslant k_{0}>0$ for every $\underline{v}_{k} \in \mathcal{V}_{I}^{h}$. We define the outflow direction $\Lambda(k)=j$ if $e(k, j) \in \mathcal{E}_{I}^{h}$ is the edge of steepest descent from $\underline{v}_{k} \in \mathcal{V}_{I}^{h}$; any one of them if such an edge is not unique. For the flux accumulation, we first set the initial values of the vertex fluxes $\widetilde{Q}_{k}=\left(\widetilde{f}, \chi_{k}\right)$ for every $\underline{v}_{k} \in \mathcal{V}_{I}^{h}$. Then, noting that water flows down the slopes of $\widetilde{W}^{0}$, we arrange the nodes in $\mathcal{V}_{I}^{h}$ so that

$$
\widetilde{W}^{0}\left(\underline{v}_{k_{1}}\right) \geqslant \widetilde{W}^{0}\left(\underline{v}_{k_{2}}\right) \geqslant \ldots \geqslant \widetilde{W}^{0}\left(\underline{v}_{k_{\mathcal{N}}}\right),
$$

where $\mathcal{N}=\# \mathcal{V}_{I}^{h}$ is the number of inner vertices, and set for $i=1, \ldots, \mathcal{N}$

$$
\widetilde{Q}_{\Lambda\left(k_{i}\right)}=\widetilde{Q}_{\Lambda\left(k_{i}\right)}+\widetilde{Q}_{k_{i}} .
$$


Finally, for $e_{k, j} \in \mathcal{E}_{B}^{h}$ we set the edge flux $Q_{e_{k, j}}=0$ and for $e_{k, j} \in \mathcal{E}_{I}^{h}$ set

$$
Q_{e_{k, j}}=\left\{\begin{aligned}
\widetilde{Q}_{k} & \Lambda(k)=j, \\
-\widetilde{Q}_{j} & \Lambda(j)=k, \\
0 & \text { otherwise. }
\end{aligned}\right.
$$

It is a simple matter to check, on noting (3.1), (3.6) and the steady state version of (3.8a), that $\underline{Q} \in \underline{Y}^{h}$, recall (4.3). Moreover, $\underline{Q} \in \underline{Y}^{h}$ solves (4.4) with $M_{\varepsilon}^{h}(W)$ replaced by $M_{0}^{h}\left(\widetilde{W}^{0}\right)$, on noting the steady state version of (3.35) and that the fluxes $Q_{e_{k, j}}$, by construction, are only nontrivial on critical edges.

We note that already on the first iteration of the lake filling algorithm, the vertices $\underline{v}_{j} \in \mathcal{V}_{I}^{h}$ connected by the edge of the steepest $\widetilde{W}^{0}$ descent to the domain boundary get their final level values, $L_{j}=\widetilde{W}_{j}^{0}$. On the second iteration vertices $\underline{v}_{k}$, whose $\widetilde{W}^{0}$ steepest descent edge path to the boundary consists of two edges, are fixed, i.e. $L_{k}=\widetilde{W}_{k}^{0}$; and so on. Therefore the total number of iterations required does not exceed the maximal number of edges in the $\widetilde{W}^{0}$ steepest descent edge path from a mesh vertex to the boundary. Our numerical experiments show that this lake filling algorithm is fast. In addition, the main part, in terms of CPU time, of the flow routing and flux accumulation algorithm is the sorting of vertices, which needs only $O(\mathcal{N} \log \mathcal{N})$ operations.

If a real depression is known, the lake filling algorithm can easily be extended to account for a partially filled closed lake. It requires only to choose a vertex, $\underline{v}_{j}$, in this depression and set initially $L_{j}=L_{0}^{\star}$, where the desired level $L_{0}^{\star} \geqslant W_{j}^{0}$ and is less than the level of a fully filled depression. The resulting profile $\widetilde{W}^{0}$ will then contain an inner lake with an almost horizontal surface at the height $L_{0}^{\star}$. Then the flow routing algorithm can be modified with a flow direction to a fictitious sink being assigned to all vertices corresponding to the remaining local minima of the relief and the edge fluxes in the lake area disregarded.

\subsection{A real relief example}

In this example we used a DEM of the Réunion island (France), which is a $63 \mathrm{~km}$ long and $45 \mathrm{~km}$ wide volcanic island in the Indian ocean. The island has a mountainous relief, see Figure 5, with its highest point about $3000 \mathrm{~m}$ above sea level. The raster DEM of the Réunion was derived from the worldwide elevation public domain database [28] collected in the "Shuttle Radar Topography Mission" project [12]. The file contained the heights above the sea level in a $72.8 \mathrm{~km}$ by $66.2 \mathrm{~km}$ rectangle $\Omega^{h}$ (Figure 5, right) at the points of a regular $809 \times 736$ grid. The horizontal resolution was thus $90 \mathrm{~m}$; the ocean points elevation was zero and the vertical DEM resolution was $1 \mathrm{~m}$.

In our numerical experiment we used a general Matlab-generated triangular mesh with $h=$ $120 \mathrm{~m}$. It contained 1,155,917 triangles, 579,118 vertices, and 1,732,717 edges. Elevations of the initial relief $W^{0}$ at the mesh vertices, $W_{j}^{0}, \underline{v}_{j} \in \mathcal{V}^{h}$, were bilinearly interpolated from the DEM data using Matlab's interp2 routine. Unlike the time consuming domain triangulation and preparation of the necessary mesh structures, the interpolation itself took less than one second.

We used $k_{0}=10^{-6}$ in the lake filling algorithm, see Section 6.1. The algorithm produced a depressionless relief $\widetilde{W}^{0}$ after 98 iterations that took 13 seconds of CPU time. The flux accumulation was computed for the uniform source $\widetilde{f} \equiv 1$, so the water fluxes obtained can be regarded as approximations to river basin areas. Together with flow routing, this computation took about 12 minutes. 

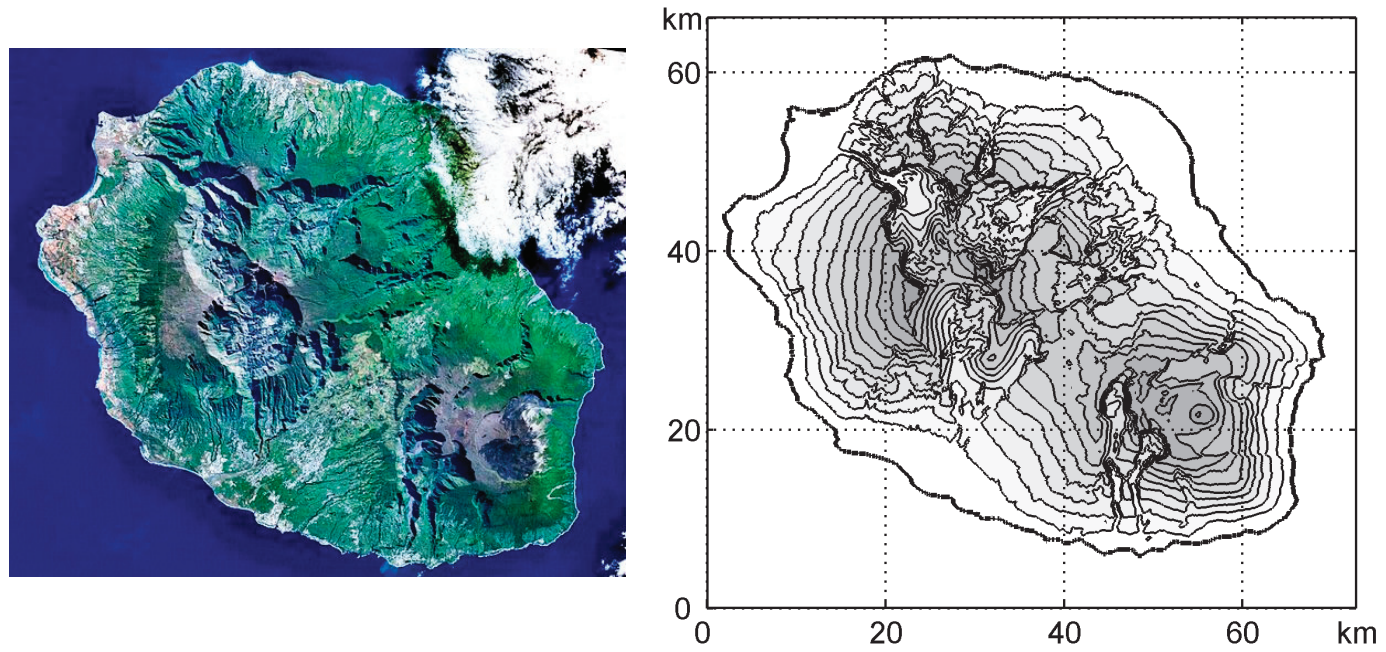

FIG. 5. Réunion island. The satellite image [29] (left) and a topographic map (right) derived using the SRTM [28] DEM employed in our simulation
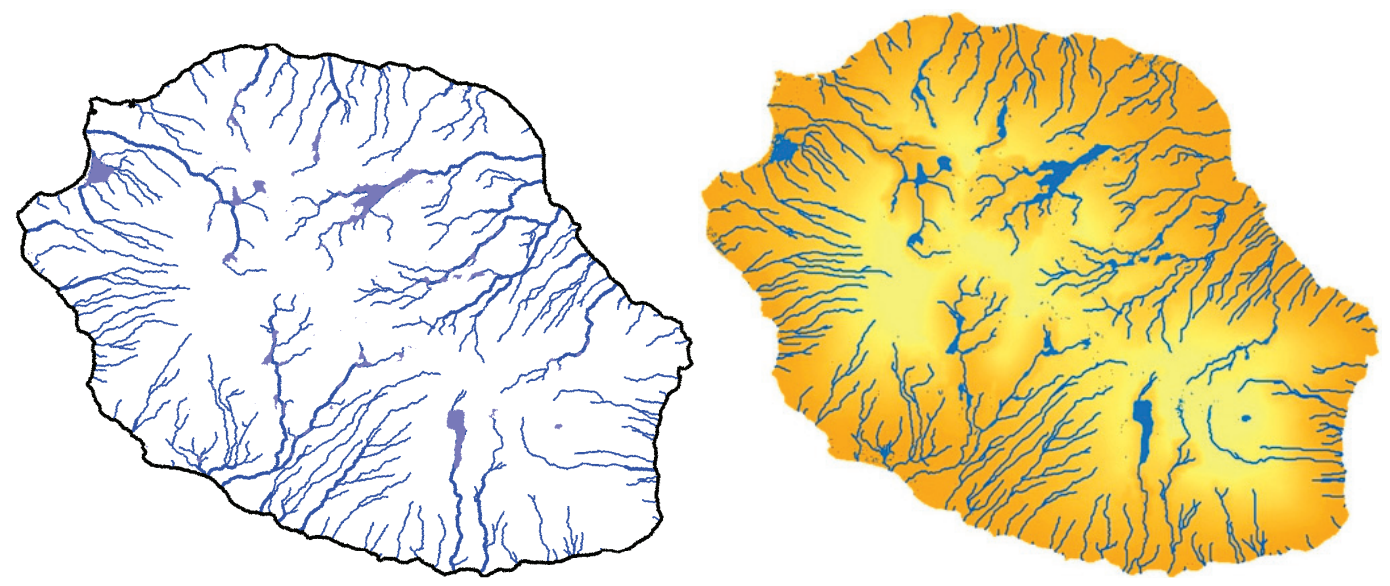

FIG. 6. DEM based hydrological maps of the Réunion island: Our simulation results (left) and the Arc Hydro [1] package map (right)

In our map of the river network (Figure 6, left) we plotted edges $e_{k, j} \in \mathcal{E}_{I}^{h}$ with the flux (drainage area) $\left|Q_{e_{k, j}}\right| \geqslant q_{0}$, where the resolution $q_{0}$ was $(1 / 2000) \int_{\Omega^{h}} \widetilde{f} \mathrm{~d} x=\left|\Omega^{h}\right| / 2000$. This resolution was adjusted to the unknown resolution of the map produced for the same DEM by the Arc Hydro [1] (Figure 6, right), based on the Jenson and Domingue algorithm [16]. The thicker lines in our map show rivers having basin areas not less than $10 q_{0}$, and the rivers are not shown in the lakes. Visually, the two maps are similar. 
Acknowledgements. The authors are grateful to Natalie Panov for her help with the DEM files and Arc Hydro.

\section{REFERENCES}

1. Arc Hydro tool of ArcGIS, esri (C).

2. Aronsson, G., Evans, L. C. \& Wu, Y., Fast/slow diffusion and growing sandpiles. J. Diff. Eqns. 131 (1996), 304-335. Zb10864.35057 MR1419017

3. BARRett, J. W. \& Prigozhin, L., Dual formulations in critical state problems. Interfaces and Free Boundaries 8 (2006), 349-370. Zb11108.35098 MR2273233

4. Barrett, J.W. \& Prigozhin, L., A mixed formulation of the Monge-Kantorovich equations. Mathematical Modelling and Numerical Analysis 41 (2007), 1041-1060. Zbl1132.35333 MR2377106

5. BARRetT, J.W. \& Prigozhin, L., A quasi-variational inequality problem arising in the modeling of growing sandpiles. Mathematical Modelling and Numerical Analysis 47 (2013), 1133-1165. Zb106198333

6. BRAUn, J. \& SAMBridge, M., Modelling landscape evolution on geological time scales: A new method based on irregular spatial discretization. Basin Research 9 (1997), 27-52.

7. Dorfman, J. \& Evans, L.C., A "lakes and rivers" heuristic metaphor for the singular limit of a nonlinear diffusion PDE. SIAM J. Math. Anal. 41 (2009), 1621-1652. Zbl1213. 35049 Mr2556577

8. Dumont, S. \& IgBida, N., On a dual formulation for the growing sandpile problem. Euro. J. Appl. Math. 20 (2009), 169-185. Zbl1158.74012 MR2491122

9. Ekeland, I. \& And Temam, R., Convex Analysis and Variational Problems (North-Holland, Amsterdam, 1976). Zb10322.90046 MR0463994

10. Elad, M., Sparse and Redundant Representations. From Theory to Applications in Signal and Image Processing (Springer, New York, 2010). Zb11211.94001 MR2677506

11. Freeman, T. G., Calculating catchment area with divergent flow based on a regular grid. Computers \& Geosciences 17 (1991), 413-422.

12. Farr, T. G., Rosen, P. A., CARo, E. Et Al., The Shuttle Radar Topography Mission. Rev. Geophys. 45 (2007), RG2004.

13. Garbrecht, J. \& MARTZ, L. W., The assignment of drainage direction over flat surfaces in raster digital elevation models. Journal of Hydrology 193 (1997), 204-213.

14. GLowinski, R., Numerical Methods for Nonlinear Variational Problems (Springer Verlag, New York, 1984). ZbI0536. 65054 MR0737005

15. Jana, R., Reshmindevi, T. V., Arun, P. S. \& Eldho, T. I., An enhanced technique in construction of the discrete drainage network from low-resolution spatial database. Computers and Geosciences, $\mathbf{3 3}$ (2007), 717-727.

16. Jenson, S. K. \& Domingue, J. O., Extracting topographic structure from digital elevation data for geographic information system analysis. Photogrammetric Eng. Remote Sensing. 54 (1988), 1593-1600

17. Jones, N. L., Wright, S. G. \& Maidment, D. R., Watershed delineation with triangle-based terrain models. J. Hydraul. Eng. 116 (1990), 1232-1251.

18. JONES, R., Algorithms for using a DEM for mapping catchment areas of stream sediment samples. Computers and Geosciences 28 (2002), 1051-1060.

19. Matlab Optimization Toolbox, ver. 6.0, The MathWorks, Inc.

20. Metz, M., Mitasova, H. \& HARMON, R. S., Efficient extraction of drainage networks from massive, radar-based elevation models with least cost path search. Hydrol. Earth Syst. Sci. 15 (2011), 667-678.

21. Nardi, F., Grimaldi, S., Santini, M., Petroselli, A. \& Ubertini, L., Hydrogeomorphic properties of simulated drainage patterns using digital elevation models: the flat area issue. Hydrological Sciences Journal 53 (2008), 1176-1193. 
22. Prigozhin, L., Quasivariational inequality describing the shape of a poured pile. Zh. Vychisl. Mat. Mat. Fiz. (in Russian) 26 (1986), 1072-1080.

23. Prigozhin, L., Sandpiles and river networks: Extended systems with nonlocal interactions. Phys. Rev. E 49 (1994), 1161-1167. MR1379784

24. Prigozhin, L., Variational model of sandpile growth. Euro. J. Appl. Math. 7 (1996), 225-235. Zb10913. 73079 MR1401168

25. Refice, A., Giachetta, E. \& Capolongo, D., SignUM: A Matlab, TIN-based landscape evolution model. Computers \& Geosciences 45 (2012) 293-303.

26. Rodrigues, J.F. \& SAntos, L., Quasivariational solutions for first order quasilinear equations with gradient constraint. Arch. Ration. Mech. Anal. 205 (2012), 493-514. Zbl1255. 49020 MR2947539

27. Rodriguez-Iturbe, I. \& Rinaldo, A., Fractal River Basins (Cambridge Univ. Press, 1997).

28. The Shuttle Radar Topography Mission (SRTM); http://www2.jpl.nasa.gov/srtm/.

29. Wikipedia, the Free Encyclopedia; http://en.wikipedia.org/wiki/Reunion.

30. Wilson, J. P., Aggett, G., Deng, Y. \& Lam, C. S., Water in the landscape: A review of contemporary flow routing algorithms. In: Advances in Digital Terrain Analysis, Lect. Notes in Geoinformation and Cartography XIV (2008), 213-236 (Eds. Q. Zhou, B. Lees, G. Tang).

31. Wilson, J. P., LAM, C. S. \& DENG, Y., Comparison of the performance of flow-routing algorithms used in GIS-based hydrological analysis. Hydrol. Process. 21 (2007), 1026-1044. 